\title{
RF wave coupling, plasma heating and characterization of induced plasma-material interactions in WEST L-mode discharges
}

\author{
G. Urbanczyk ${ }^{\mathrm{a}}$, L. Colas ${ }^{\mathrm{b}}$, J. Hillairet ${ }^{\mathrm{b}}$, E. Lerche ${ }^{\mathrm{e}}$, N. Fedorczak ${ }^{\mathrm{b}}$, J. Morales $^{\mathrm{b}}$, \\ J. Gunn ${ }^{\text {b }}$, V.Ostuni ${ }^{\mathrm{b}}$, S. Heuraux ${ }^{\mathrm{c}}$, D. Vezinet ${ }^{\mathrm{b}}$, V. Bobkov ${ }^{\mathrm{d}}$, C. Desgranges ${ }^{\mathrm{b}}$, A. Ekedahl ${ }^{\mathrm{b}}$, \\ P. Mollard ${ }^{\mathrm{b}}$, G. Lombard ${ }^{\mathrm{b}}$, B. Pégourié ${ }^{\mathrm{b}}$, T. Loarer ${ }^{\mathrm{b}}$, W. Helou ${ }^{\mathrm{f}}$, C.C. Klepper ${ }^{\mathrm{g}}$,

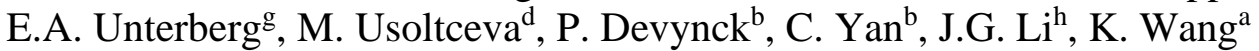 \\ and WEST Team (http://west.cea.fr/WESTteam) \\ ${ }^{a}$ Key Laboratory of Optoelectronic Devices and Systems, College of Physics and Optoelectronic Engineering, \\ Shenzhen University, Shenzhen 518060, China \\ ${ }^{b}$ CEA, IRFM, F-13108 Saint Paul-Lez-Durance, France. \\ ${ }^{c}$ Institut Jean Lamour, Université de Lorraine-CNRS F-54000 Nancy, France \\ ${ }^{d}$ Max-Planck-Institut für Plasmaphysik, Boltzmannstrasse 2,D-85748 Garching, Germany \\ ${ }^{e}$ Laboratorium voor Plasmafysica - Laboratoire de Physique des Plasmas, Association 'EURATOM - \\ Belgian State', Trilateral Euregio Cluster, 30 Avenue de la Renaissance, B-1000, Brussels, Belgium \\ ${ }^{f}$ ITER Organization, Route de Vinon-sur-Verdon, CS 90 046, 13067 St. Paul, Lez Durance Cedex, France \\ ${ }^{g}$ Oak Ridge National Laboratory, Oak Ridge, TN, United States of America \\ ${ }^{h}$ Institute of Plasma Physics, Chinese Academy of Sciences, Hefei 230031, People's Republic of China
}

guiguiurban@hotmail.com, taiyang.lin@ipp.ac.cn

\begin{abstract}
Plasma heating in the full Tungsten (W) Environment in Steady-state Tokamak (WEST) relies on electromagnetic waves in both Lower Hybrid (LH) and Ion Cyclotron Range of Frequencies (ICRF). The present study focuses mostly on the optimization of discharges heated with ICRF, by reporting different methods to first optimize wave coupling, optimize their absorption and reduce the impurity production.

It is shown that ICRF coupling can be optimized either by moving the plasma closer to antennas, increasing the plasma density, wave frequency and LH power. We show that the absorption efficiency correlates with the hydrogen concentration with the existence of an optimum between 7 and $10 \%$ as expected for a minority heating scenario.

Absolutely calibrated visible spectroscopy sightlines were used to monitor ion fluxes in different locations as part of an effort to quantitively estimate the contribution of different impurity sources to the core contamination by tungsten. It is typically found that in discharges with high total RF-power (above $5 \mathrm{MW}$ of $\mathrm{LH}$ and $3 \mathrm{MW}$ of ICRF), divertor and antenna limiter sources can reach similar order of magnitude during the ICRF phase.
\end{abstract}

\section{Introduction}

The "tungsten Environment in Steady-state" Tokamak named WEST results from the transformation of the Tore Supra tokamak from a carbon limiter to a tungsten (W) divertor 
configuration. It has been designed to carry out a scientific program focusing on the preparation of ITER experiments, in particular, to test ITER-like actively cooled full tungsten Plasma-Facing Units (PFUs) [1]. The additional heating and current drive powers are provided by high-frequency heating systems, namely lon Cyclotron Range of Frequencies (ICRF) and Lower Hybrid Current Drive (LHCD), respectively composed of three identical ICRF antennas designed to deliver up to $9 \mathrm{MW}$ in total and two $\mathrm{LH}$ launchers delivering up to $7 \mathrm{MW}$ in total. Accessing heat flux levels required to validate plasmafacing units (PFUs) $\left(10-20 \mathrm{MW} / \mathrm{m}^{2}\right.$ [2]) implies coupling high power RF waves in the range of 6-7 MW. However, ICRF waves are foreseen to have a strong impact on impurities production due to RadioFrequency (RF) sheaths [3]. The development of high power scenarios requires to localize the main sources of impurities and to mitigate them when possible. While boronizations help reducing the fraction of radiated power - likely by mitigating low- $Z$ impurities such as oxygen and/or chlorine which facilitates the plasma start-up phase, this effect usually only lasts for about a few discharges. In Alcator C-Mod, high potentials were registered in regions magnetically connected to ICRF antenna and boronization assisted by ECRH was then used to locally apply low-Z coating in most problematic regions [4]. Visible spectroscopy was used to track impurities sources and influx while X-ray spectroscopy was used to detect them inside core plasma, together with allowing the definition of a penetration factor. In regions connected to Alcator C-Mod ICRF antennas and some parts of the outer divertor, the erosion of the boron layer occurred over tens of seconds in ICRF-heated $\mathrm{H}$-modes [5]. This suggests that weekly boronizations in WEST can hardly be enough to sustainably prevent RF-enhanced erosion of tungsten (typically 15-20nm/s above 1MW ICRF power [6]). In presence of ICRF, similar observations were made on ASDEX Upgrade (AUG) [7] while boronization effects were already studied in a full tungsten environment and the outer divertor target identified as the strongest source of impurities [8]. Similar results were found in EAST, with a large contribution from limiters to core contamination yet dominated by divertor which surface is much larger than the one of limiters [9]. In JET with ITER-like walls, the use of beryllium as plasma-facing component (PFCs) together with long machine conditioning with glows and large power from Neutral Beam Injectors, boronization is not necessary. Similar observations were made concerning stronger impurities production nearby antennas and outer divertor [10]. Erosion-redeposition modelling of edge beryllium line spectroscopy of localized, ICRF sheath-enhanced emission at magnetically connected JET outer limiters was also shown to be consistent with increased local erosion [11, 12]. An excellent overview of ICRF-induced plasma surface interaction is provided by [13], while a discussion about tungsten management for H-mode scenario's optimization in JET can be found under [14].

Among numerous 2D mapping experiments based on SOL parameters scan at antennas surrounding using various diagnostics like Retarding Field Analyzer [15], Langmuir probes [16], Lithium beam [17], Gas puff imaging [18] or Infrared Camera (IR) [3] some global trends are common to many physical values, suggesting RF-sheath somehow modifies several parameters with a reproducible fashion.

In the first section, the set of diagnostics is detailed with a description of the Ultraviolet (UV) spectroscopy, Langmuir probes, bolometry, interferometry [19], Electron Cyclotron Emission (ECE), neutron detector. The second section describes the ICRF system of WEST while insights for optimizing wave phasing, coupling and absorption in the plasma will be given in the third section. In the last section, we will specifically tackle the plasma surface interaction in different locations in WEST, essentially comparing sources on antennas limiters vs divertor. We will successively investigate various causes for plasma surface interaction, namely the radial distance between the plasma and the first metallic objects, the influence of the LH power alone, the influence of the ICRF power alone, and finally the influence of both combined together at high power level, particularly relevant for triggering transition into high confinement mode for significantly improving performances; a scenario of high interest for present and mostly future devices like ITER and the Chinese Fusion Engineering Test Reactor (CFETR). We will finally conclude and propose solutions to mitigate them. 


\section{Presentation of WEST}

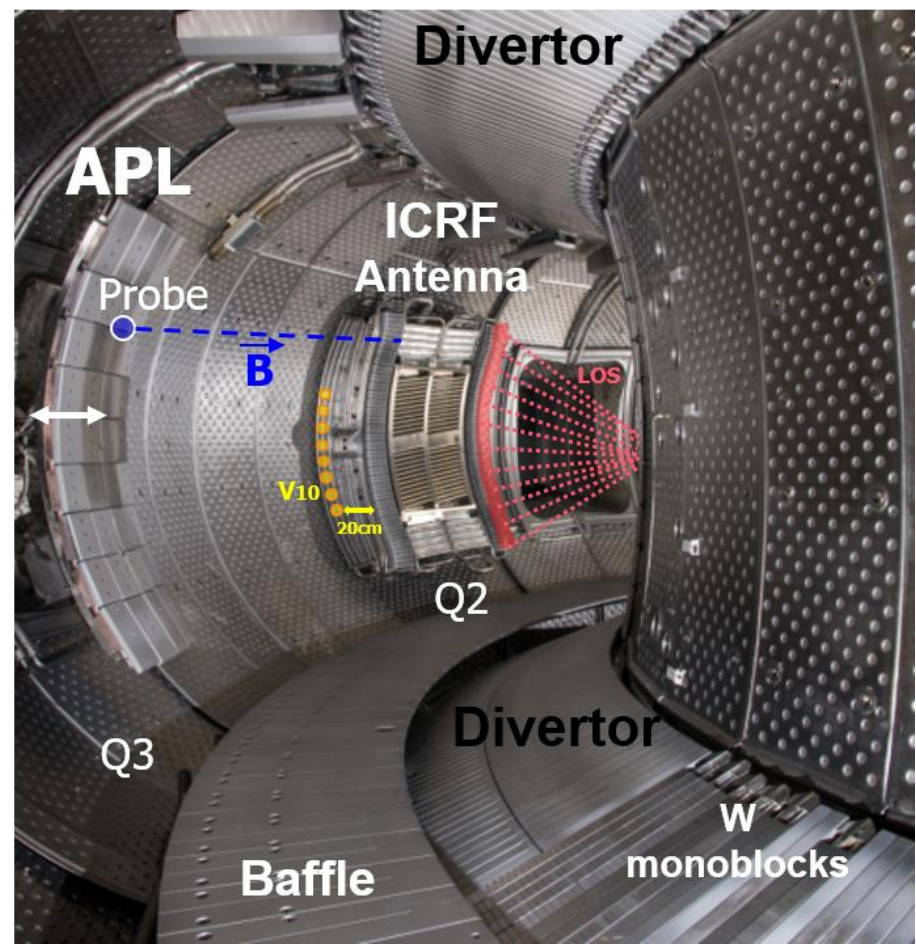

FIG 1. Picture of WEST inner vessel, representing one field line (blues dotted $B_{0}$ ) connecting the upper Pecker probe on the Antenna Protection Limiter (APL) to the upper left corner of the Q2 ICRF antenna, poloidally distributed valve V10 for plasma fueling, Lines of Sight (red dotted LOS) of the visible spectroscope which fibre bundle is embedded in the high field side wall, the baffle, the divertor with a majority of tungsten coated graphite tiles and 12 of them with ITER-like tungsten monoblocks

\subsection{General description}

WEST is a fully metallic (mostly W) Tokamak designed for operating in lower single null, upper single null or double null configurations with typical parameters $B_{T}=3.7 T$ at $R_{0}=2.5 \mathrm{~m}, a=0.5 \mathrm{~m}$ and $I_{p} \approx 500 \mathrm{kA}$. Its main goal is to test and validate the tungsten monoblocks PFUs design for ITER divertor with $10 \mathrm{MW} / \mathrm{m}^{2}$ in steady-state and up to $20 \mathrm{MW} / \mathrm{m}^{2}$ for transient events (ELMs) [20]. The auxiliary heating system is composed of two Lower Hybrid Current Drive (LHCD) and three ICRF antennas [21]. The LHCD system is composed of two antennas powering respectively $4 \mathrm{MW}$ for the Full Active Multijunction (FAM) launcher [22] and $3 \mathrm{MW}$ for the Passive Active Multijunction (PAM) launcher [23]. The purpose of the LHCD system is not only to heat the plasma, but also to non-inductively drive current and shape its profile to allow steady-state discharge up to about 1000s. The ICRF system is the main heating system, expected to deliver once fully commissioned up to $9 \mathrm{MW}$ for 30s, $6 \mathrm{MW}$ for $60 \mathrm{~s}$ and $3 \mathrm{MW}$ for up to 1000 s operating generally at $55.5 \mathrm{MHz}$. Further details about the ICRF system are given in the next section. All antennas (LHCD and ICRF) are actively cooled allowing for long pulse operation whilst gas can also be injected from different valves either at the top, or near antennas limiters to try to increase the density at the edge and improve the wave coupling [24, 25]. Gas can be injected from over ten different valves but the one that will be used for the present study are essentially materialized by yellow circles in Fig.2a, respectively the top valve (V11) and near limiters at the low field side, either form a single point located at the mid-plane (V7 on APL) or poloidally distributed along with the object's poloidal height (ex: V9, V10). 

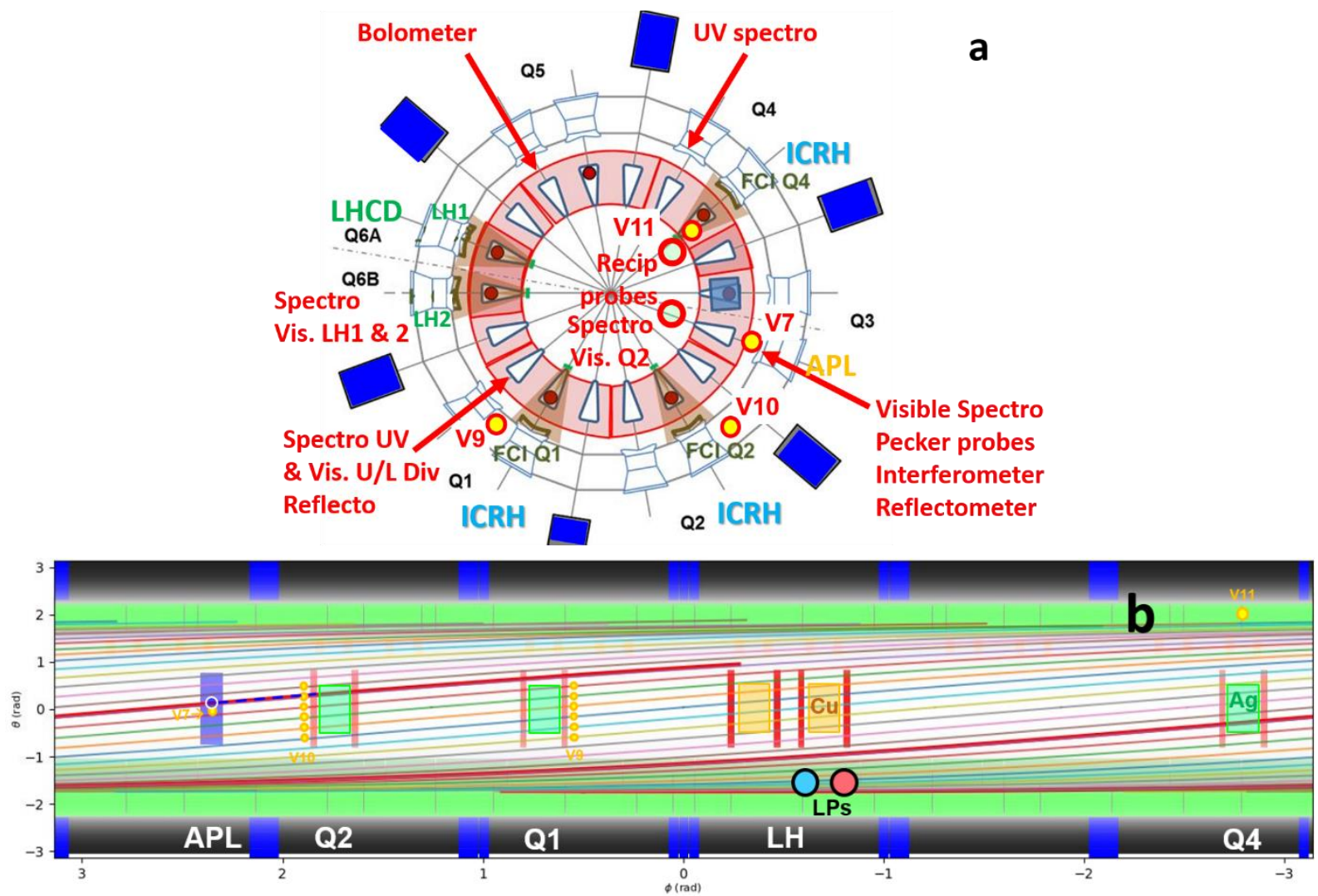

FIG 2. WEST (a) Top view with diagnostics of interest for the study, two LH launchers and three ICRF antennas, and fueling valves with top (V11) poloidally distributed (V10) and nozzle valves at midplane

(V7) and (b) Field lines tracing (seen from inside of the torus) for a typical discharge (\#55713) with $\mathrm{Bt}=3.7 \mathrm{~T}$ and $\mathrm{Ip}=500 \mathrm{kA}$, the thick line showing the possible magnetic connection of the upper pecker probe on the APL. The blue dot represents the position of the Langmuir probes array in the outer target $315.4^{\circ}=-0.78 \mathrm{rad}$ and the red one on the inner target $324.9^{\circ}=-0.6 \mathrm{rad}$.

\subsection{Diagnostics and data interpretation}

Among preferential diagnostics used in this study, WEST is equipped with Electron Cyclotron Emission (ECE) diagnostic with 32 channels along the equatorial plane to measure electron temperature profiles. The radial resolution $\left(\delta R^{\sim} 2.4 \mathrm{~cm}\right)$ is essentially limited by relativistic effects, depending on electron temperature and density, and not by the channels' frequency spacing, while pedestal measurements are often difficult due to the presence of LHCD-induced supra-thermal electron emission that tends to pollute measurement for $r / a>0.8$. By default, the time resolution is set to $1 \mathrm{~ms}$, but can also be decreased down to $1 \mu \mathrm{s}$ for studying MHD events.

The power radiated is measured along 16 bolometry chords crossing the plasma at different poloidal heights leading to $9 \mathrm{~cm}$ spatial resolution near the separatrix and $8 \mathrm{~ms}$ time resolution.

Two extreme UV spectrometers with a central line allow measuring uncalibrated brightness emitted from metal impurities in the core plasma, with wavelengths respectively ranging from 0.1 to $70 \mathrm{~nm}$ and 22 to $30 \mathrm{~nm}$ [26]. The latter is also used for controlling impurity content in the plasma in real-time, and serve as a safety system in case of critical plasma surface interaction. For instance, when the intensity of $\mathrm{Cu}^{18+}$ overcomes a reference value, the RF power is automatically lowered or switched off as it may be the result of deleterious interactions on the LH launchers. Another VUV spectrometer $(0.1-70 \mathrm{~nm})$ can specifically monitor either the quasi-continuum emitted by $\mathrm{W}^{27+}-\mathrm{W}^{35+}$ or spectral lines emitted by $\mathrm{W}^{38+}-\mathrm{W}^{45+}[27]$.

In addition to these core diagnostics, the edge plasma is monitored by diagnostics such as probes and visible spectroscopy. Some of the probes are flush-mounted along with the divertor 
targets [28], while some are reciprocating [29] either from the APL (Fig.1) or from the top of the device, with design immune to sheath expansion [30], therefore favourable for measurements in RF environment.

The edge visible spectroscopy was deployed primarily in the near-UV to the blue spectral region $(360-420 \mathrm{~nm})$ to assess the spectral region of interest for studying $W$ sources. The prominent neutral and singly ionized $W$ emission line $(400.8 \mathrm{~nm})$ fall in this spectral region [31]. 52 lines of sights (LOS) can monitor among 240 different locations both on some antenna limiters $(86 \mathrm{~mm}$ spatial resolution or 12 along with the poloidal height of each limiter) and divertor (10 $\mathrm{mm}$ spatial resolution) [32]. For the campaigns presented in this study though, LOS were not yet available to monitor the Q1 and Q4 ICRF antennas, so that we will only show data measured on Q2 antenna limiters. Two spectroscopes, with 27 lines each, are used simultaneously to locally follow neutral state of tungsten. We have therefore used what was learnt from previous experiments to prioritize regions to monitor, to the eventual detriment of hidden impurity sources like in JET [10,33]. By order of importance based on literature, it was chosen to look at some limiters of active antennas (essentially the right limiter of Q2 antenna as shown in Fig.1) and divertor targets. Others objects were also monitored, but will not be discussed as the intensity of these sources usually fall several order of magnitude below the ones on antenna limiters and divertor. For instance, the Antenna Protection Limiter (APL), despite partial magnetic connections to the Q2 and Q4 ICRF antennas as shown in Fig.2b, is radially retracted about $2 \mathrm{~cm}$ behind antenna limiters during the discharges, falling in a region of lower electron temperature and density, by then becoming a negligible source. This is also the case of the baffle which the leading edge in general sits pretty far from the separatrix, and the inner bumper at the high field side wall.

Another spectrometer with higher spectral resolution allows focusing on $\mathrm{D} \alpha$ and $\mathrm{H} \alpha$ lines of Balmer series for measuring hydrogen concentration (further discussed in section 3.3).

$\mathrm{W}$ outflux is approximated as the sum of each ion influx on tungsten materials, pondered by their respective sputtering yields $\mathrm{Y}_{\mathrm{s}}$ (probability to sputter an atom of tungsten) :

$$
\Gamma_{W}^{\text {out }}=\Gamma_{D} \cdot Y_{D}+\Gamma_{\text {Low }-Z} \cdot Y_{\text {Low }-Z}+\Gamma_{W}^{\text {in }} \cdot Y_{W}
$$

With $\Gamma_{W}^{\text {in }}$ and $\Gamma_{W}^{\text {out }}$ resp. the tungsten influx and outflux, $\Gamma_{D}$ and $\Gamma_{L o w-Z}$ the deuterium and low-Z particles influx (ex: $\mathrm{O}, \mathrm{C}$ ) and their respective sputtering yields $Y_{D}$ and $Y_{\text {Low-Z }}$. Assuming that the deuterium flux is representative of all particle fluxes regardless of the plasma composition, it is possible to experimentally estimate the average efficiency of an ion to sputter a W atom, this is the so-called effective sputtering yield $Y_{\text {eff }}$ :

$$
\frac{\Gamma_{W}^{\text {out }}}{\Gamma_{D}}=Y_{D}+\frac{\Gamma_{L o w-Z}}{\Gamma_{D}} \cdot Y_{L o w-Z}+\frac{\Gamma_{W}^{i n}}{\Gamma_{D}} \cdot Y_{W}=Y_{E f f}
$$

The determination of the flux of a given species $\Gamma_{\mathrm{s}}$ is assessed from the integral of corresponding lines in the spectra multiplied by the so-called $S / X B\left(T_{e}, n_{e}\right)$ coefficient. $S / X B$ is a spectroscopic parameter converting emission line intensity into an influx of impurity atoms from limiting surfaces, i.e., the ionization (S) per excitation rate $(\mathrm{X})$ corrected for the branching ratio (B). It can be assessed from ADAS database [34, 35].

Since the divertor target is equipped with Langmuir probes [36], it will be possible to locally determine the value of these coefficients. When looking at antenna limiters, however, no diagnostic systematically measures local $T_{e}$ nor $n_{e}$, only pecker probes can plunge about twice a shot from the APL magnetically connected to the limiters of the Q2 ICRF antenna (cf. blue lines in Fig.1 and Fig.2b). We will therefore assume that these parameters are not changing much, following what was observed in Tore Supra [37] and other devices like Alcator C-Mod [4] and ASDEX Upgrade [7]. With typically measured temperatures and densities of about $T_{e}=15 \mathrm{eV}$ and $n_{e}=10^{17} \mathrm{~m}^{-3}$, it leads to $\mathrm{S} / \mathrm{XB}$ of the order of 20 for $W, 10$ for $D_{\alpha}(656.1 \mathrm{~nm}), 2000$ for $D_{\delta}(410.2 \mathrm{~nm})$ and 7950 for $D_{\varepsilon}(397 \mathrm{~nm})$. We will therefore estimate the effective sputtering yields from photon fluxes $\left(\mathrm{DI}_{\delta}\right.$ and $\mathrm{WI}$ respectively neutral deuterium (from Balmer series) and tungsten) as follow: 


$$
Y_{E f f}=\frac{\Gamma_{W}}{\Gamma_{D}}=\frac{W I}{D I_{\text {Balmer }}} \cdot \frac{S / X B_{W I}\left(T_{e}\right)}{S / X B_{D I}\left(T_{e}\right)}
$$

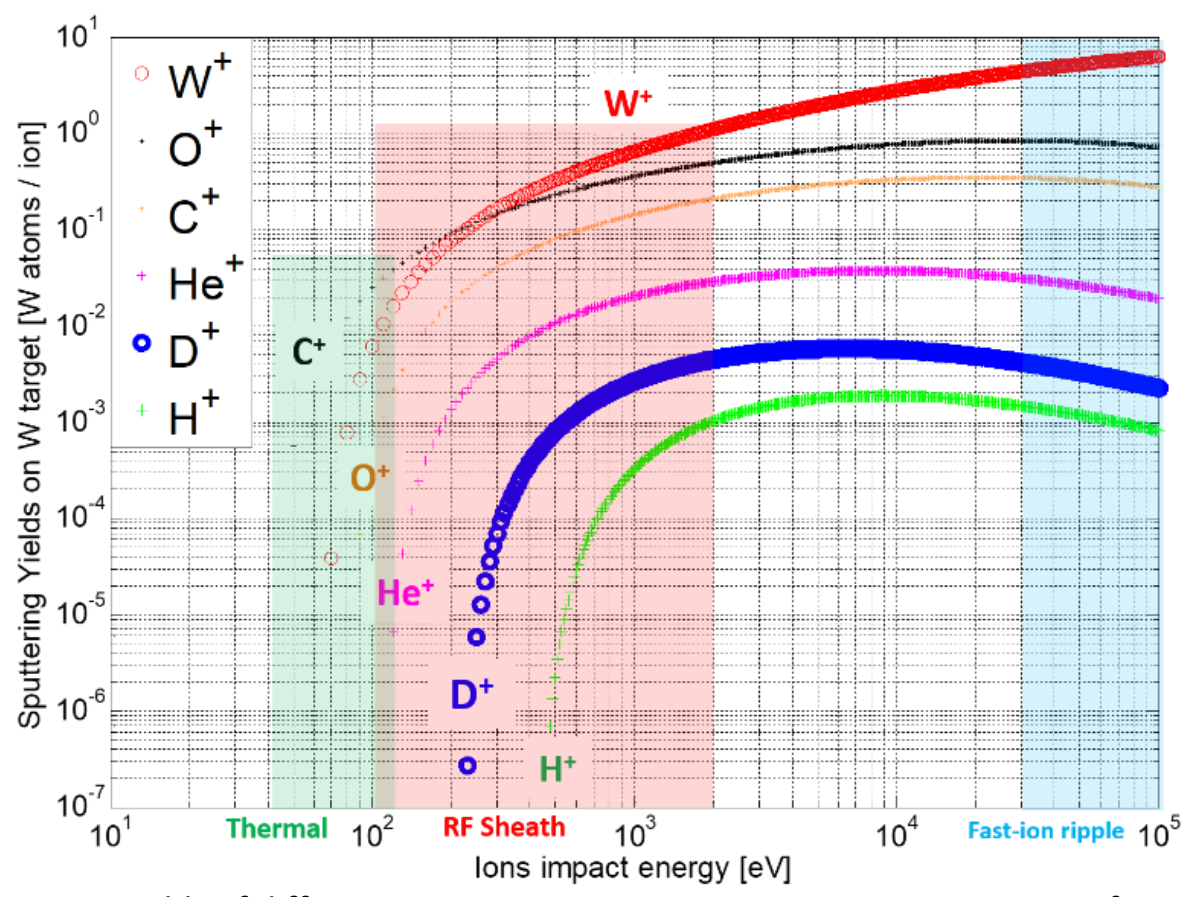

FIG 3. Sputtering yields of different ion species present in WEST on W target as a function of ions impact energy. Green, red and blue regions correspond respectively to incident particle energy ranges related to thermal sheath (linear $\sim 3 \mathrm{k}_{\mathrm{B}} \mathrm{T}_{\mathrm{e}}$ ) [38], RF sheath (non-linear) and fast-ion ripple losses [39].

Despite S/XB coefficients do not vary much in the SOL, Fig,3 shows that the effective sputtering yield also depends on the type of incident ion. Basically, the higher the weight and the ionization state, the most detrimental. In the red region - corresponding to the range of energy of ions typically accelerated through the sheath in presence of ICRF $[15,37,40]$ - sputtering yields tend to increase very sharply and non-linearly. RF-sheath is therefore a mechanism that can enhance erosion by several orders of magnitude. However, without ICRF, the potential drop across the sheath is usually much smaller (cf. green region in Fig.3) such that most ions reach the wall with little energy and are not very efficient in sputtering atoms. The temperature threshold is typically in the range of $40 \mathrm{eV}$ because of the Maxwellian tail, further shifted by the sheath. This is why the levels of impurity production in plasmas only heated by LHCD remain small.

Finally, we can assess the influence of the sputtering by $\mathrm{H}^{+}$ions lost in ripples during ICRF in the minority heating scheme, considering $10 \%$ of the ICRF power lost in ripple [39]. For about $1 \mathrm{MW}$ ICRF power, $100 \mathrm{~kW}$ power is therefore lost into ripple by $\mathrm{H}^{+}$ions with about $100 \mathrm{keV}$ energy. This represents a hydrogen influx of about $\Gamma_{\mathrm{H}+}=10^{19}$ ions/s, which multiplied by the sputtering yield of $\mathrm{H}^{+}$ ions on tungsten $\left(\sim 10^{-3}\right.$ as seen on the green curve of Fig. 3 in the blue region) results in a tungsten atoms outflux from lower tungsten surfaces of about $\Gamma_{\mathrm{W}_{+}}=10^{16}$ ions/s. This value is about an order of magnitude lower than typical impurity sources as it will be shown in the last section. It is therefore expected not to play a significant role in the production of impurity.

In the rest of the paper, we will average fluxes along periods of time and take into account the surface of the spots of each line of sight to represent absolute particle fluxes in all figures $\left(\mathrm{atm} / \mathrm{m}^{2}\right)$. 


\section{WEST ICRF system}

The WEST ICRF system is composed of three identical antennas respectively in horizontal ports in torus quadrants Q1, Q2 and Q4 (Fig.1 and Fig.2) to which we will refer for naming each antenna. Each antenna is composed of four straps inside a box and behind a Faraday screen [41]. The Faraday Screen, the straps and the antenna box are silver coated. On each side, CFC limiters coated with $80 \mu \mathrm{m}$ of molybdenum first followed with a $80 \mu \mathrm{m}$ layer of tungsten on top (Fig.1), are protruding by $2 \mathrm{~cm}$ to protect the antenna front face from the plasma. Each pair of straps is fed by the same RF power generator whose power is divided by a conjugate-T junction to allow good load resilience [Helou 2020]. Further details concerning the RF design can be found under [41,42]. In our study, the system is operated at $55.5 \mathrm{MHz}$ for $\mathrm{H}$-minority heating.

In nominal situation, antennas are operated in dipole phasing $\left(180^{\circ}\right.$ as represented in Fig.6) which spectrum represented in Fig.4a is centred on the parallel wave vector $\boldsymbol{k}_{/ /}=11 \mathrm{rad} / \mathrm{m}$. This is not only because high $\boldsymbol{k}_{/ /}$spectra usually result in better absorption efficiencies, but also because this is the phasing that minimizes the excitation of RF-sheath that can have a deleterious influence on plasma surface interaction $[13,43]$ which is shown in the next section. However, since commissioning several systems simultaneously required setting priorities, efforts focused on matching optimization, to the detriment of accurate phasing control up to summer 2019. Fig.4b shows the properties of waves with different $\boldsymbol{k}_{/ /}$corresponding to different phasing that have occurred during several discharges before summer 2019, which calculation details can be found under [44]. It can be seen that reducing the phasing difference between the straps generally results in an attractive increase of wave coupling efficiency (efficiency by which the waves are coupled to the plasma detailed in the next section), to the cost of increasing plasma surface interaction as shown in the next section.

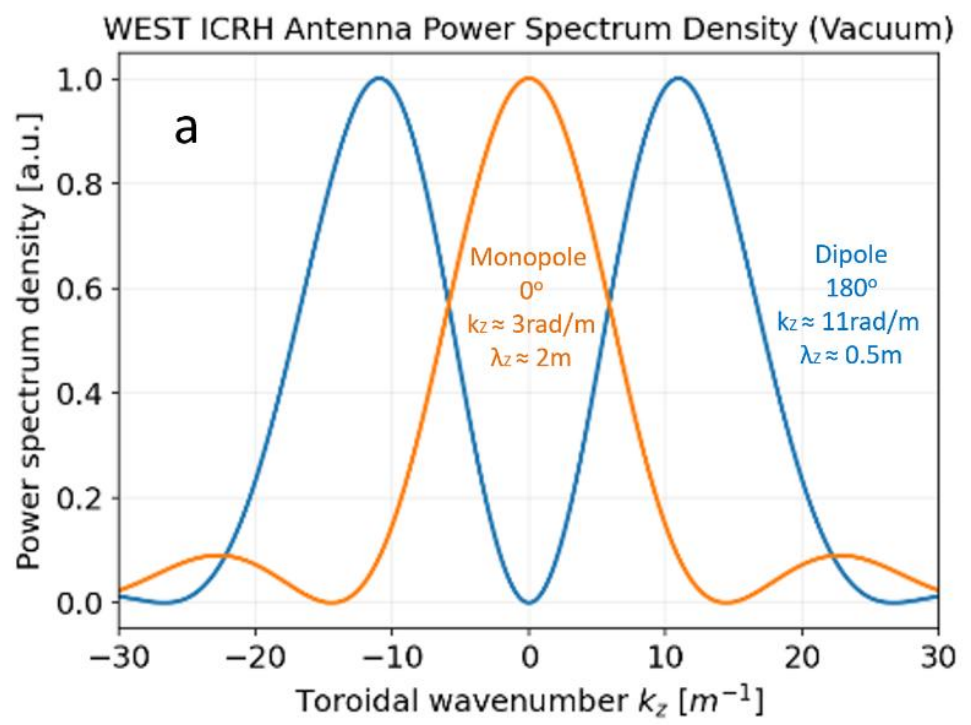




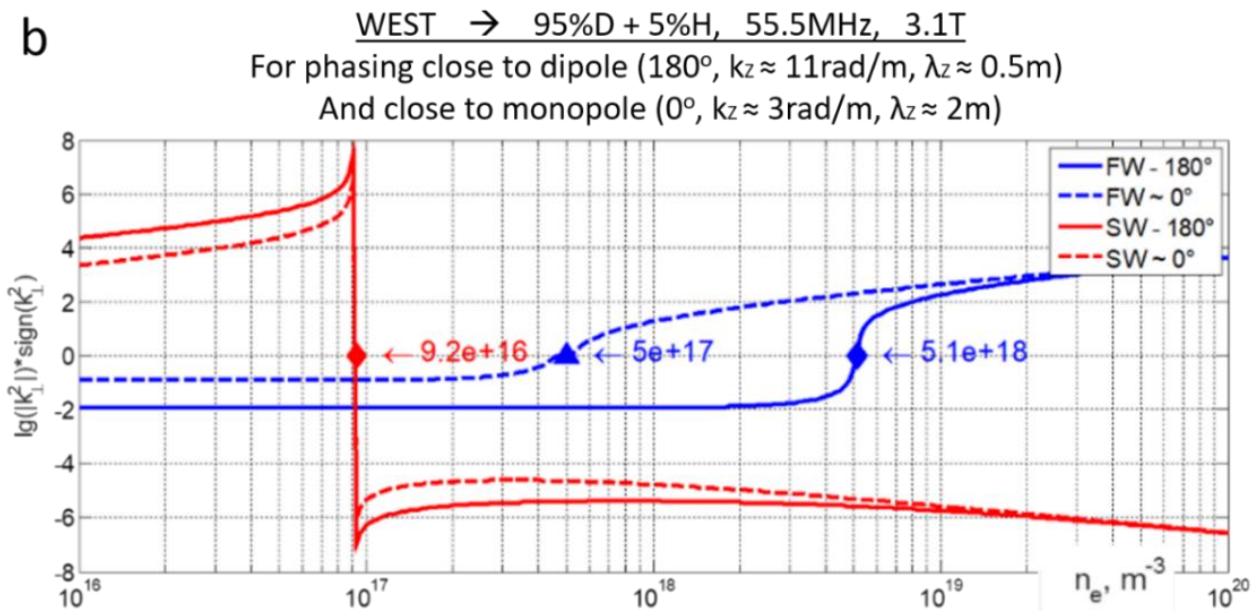

FIG 4. Calculations based on WEST ICRF antennas geometry of (a) power spectrum density in vacuum and (b) squared perpendicular wavenumbers (log scale) vs edge density in front of WEST ICRF antennas, enlightening the propagation properties of the so-called fast (blue) and slow (red) waves emitted at $55.5 \mathrm{MHz}$ in dipole $\left(180^{\circ}\right.$ - solid lines $\left.11 \mathrm{~m}^{-1}\right)$ and monopole $\left(0^{\circ}\right.$ - dotted lines) phasing in a plasma with $95 \%$ of deuterium and $5 \%$ of hydrogen. When values of the $\mathrm{Y}$-axis are positive, the wave is propagative, otherwise, it is evanescent.

\section{Parametric optimization of ICRF operation}

\subsection{ICRF phasing}

As shown in the previous section - which simple description does not take into account spectrum width -working in monopole phasing $\left(0^{\circ}\right)$ allows improving antenna loading by significantly lowering the cut-off density of the fast wave. However, in this section, we will now show that low $\boldsymbol{k}_{\| /}$ spectra can not only sometimes lower wave absorption efficiency but also lead to strong RF-sheath excitation, and therefore the increase of impurity sources $[13,43]$. The phasing between each toroidal pair of straps of Q1 antenna was scanned in the range of 70-250 degrees (Fig.5a) during the 2019 autumn campaign, by preserving similar forward power. Note the matching was not modified along with the scan but remained the one optimized for dipole phasing. Therefore, as phase changes, so does the coupling and the coupled power and voltages inside the antenna, which can significantly complicate the analysis. In the case analysed in the present study though, the change in coupled power over the phase scan remains below $5 \%$.

When the phasing passes by $180^{\circ}$ around $6.2 \mathrm{~s}$, the temperature and neutrons (Fig.5c) consistently reach their maximum. Almost at the same time, the radiated power (Fig.5a and Fig.6) also reaches its minimum. Just after, as phasing keeps shifting away from the dipole case, more and more power is radiated.

We now wish to attempt to determine the cause of such radiation increase. Firstly, looking with VUV spectroscopes at various impurities in the core, we find that the normalized brightness - by the plasma density (Fig.5d) - of both silver $\left(\mathrm{Ag}^{35+}\right)$ (from antenna Faraday Screen) and copper $\left(\mathrm{Cu}^{18+}\right)$ (presumably from magnetically connected LH launchers) increase in correlation with the radiated power (Fig.5e). Secondly, looking at tungsten brightness along with ICRF antenna limiter with visible spectroscopy lines (Fig.6), we find that the brightness - mostly at the top half - of the antenna limiter, also well match the variations observed on the radiated power. Note the up-down asymmetry of the level of tungsten, higher for $Z>0$ than for $Z<0$. This is a consequence of the plasma equilibrium which barycenter stands above $Z=0$, leading to smaller outer gaps at the upper part than at the bottom part of the antennas (cf. Fig.A2 in Appendix, also consistent with the poloidal shape of deuterium fluxes on the limiter, always peaking at $Z>0$ in Figs.12-14.a of the last section of this paper) 
This discharge, therefore, confirms the fact that ICRF antennas should better work in dipole phasing to optimize global performances (wave coupling efficiency, radiated power, electron temperature, neutrons generation and possibly impurity production) [45].

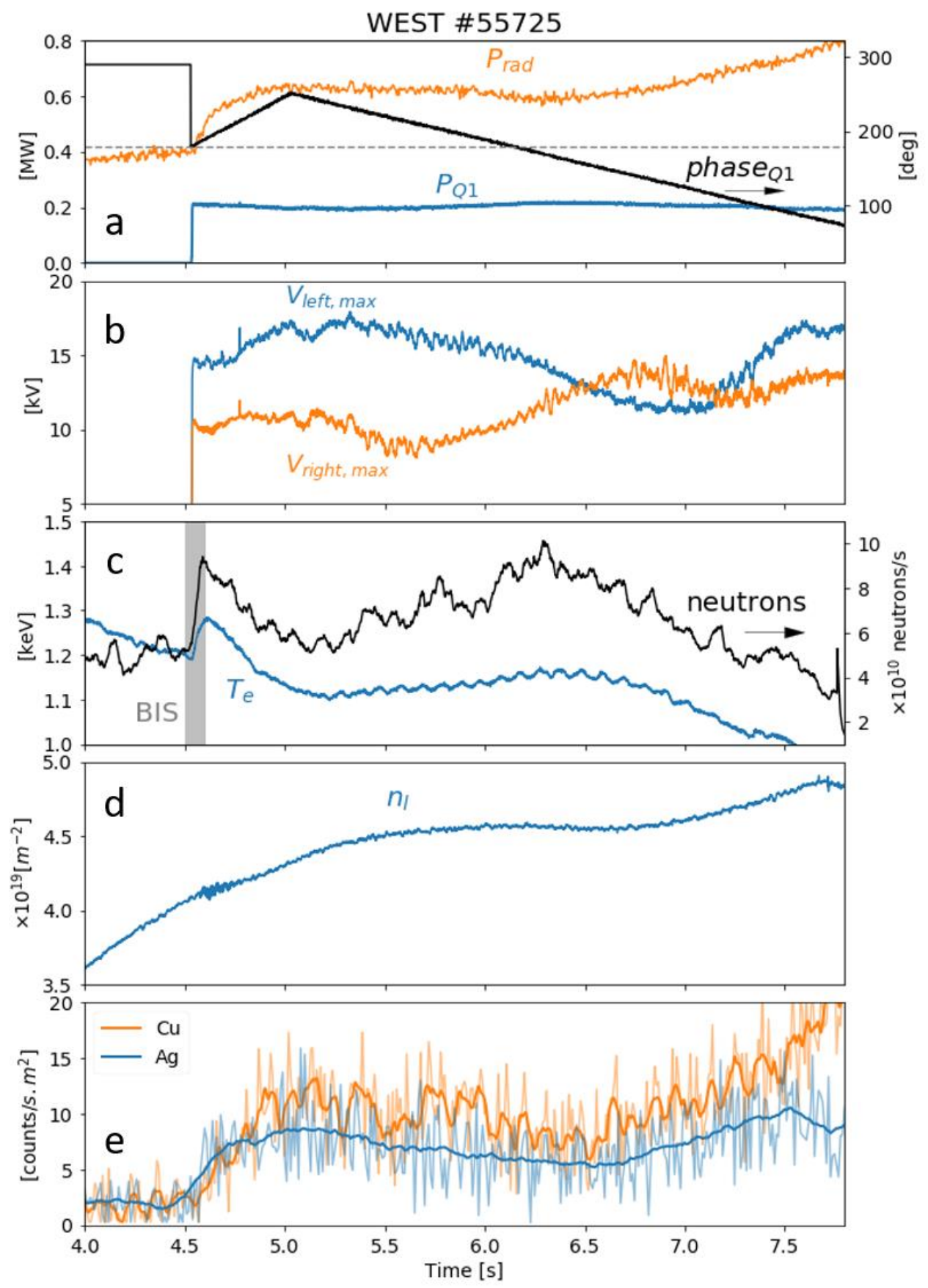

FIG 5. Time traces along Q1 antenna phasing scan during discharge 55725 of (a) radiated power and Q1 antenna coupled power, phasing and (b) voltages measured on each side of the antenna (c) electron temperature from ECE central chord and neutrons production (d) electron density integrated along central interferometry line and $(e)$ silver $\left(\mathrm{Ag}^{35+}\right)$ and copper $\left(\mathrm{Cu}^{18+}\right)$ brightness measured by VUV spectroscopes and normalized by the density. 


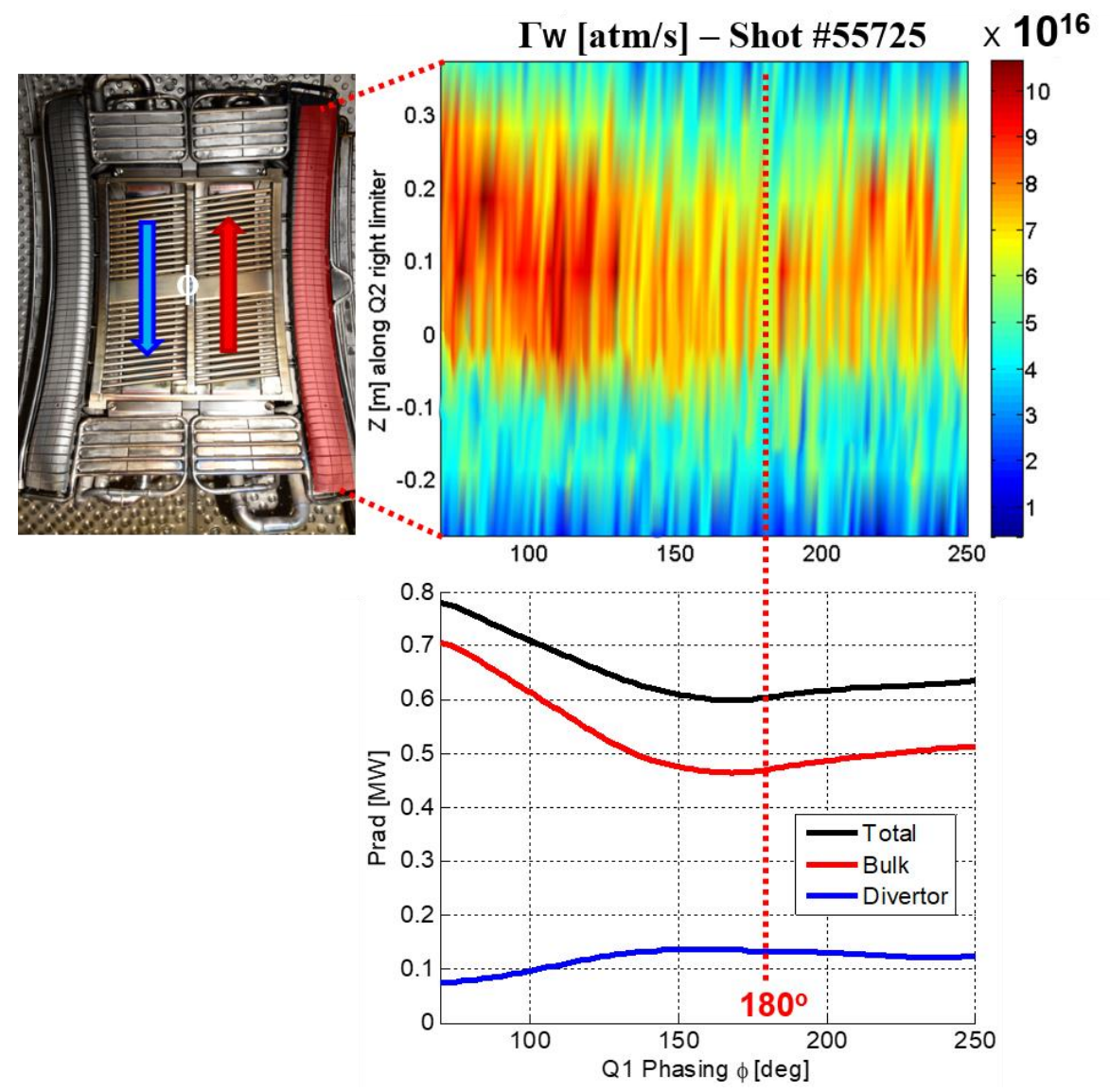

FIG 6. Evolution along Q1 antenna phase scan of the tungsten outflux measured along Q2 right side limiter poloidal height and the power radiated in the bulk, divertor and their sum.

\subsection{ICRF wave coupling}

The coupling resistance used for a half-WEST ICRF antenna $\left(R_{c}\right)$ is defined as in [42],

$$
R_{c}=\frac{2 P_{t}}{I_{s}^{2}}
$$

With

$$
I_{s}=\frac{1}{2} \sum\left|I_{i}\right|^{2}
$$

where $\mathrm{P}_{\mathrm{t}}$ is the power radiated by the antenna into the vessel and $\mathrm{I}_{\mathrm{i}}$ the currents exciting the straps array, respectively measured in WEST by bidirectional couplers and voltage probes. If the coupling is very poor (or the coupling resistances low), large RF currents or voltage can rapidly arise in the antenna. Risks of deterioration such as arcing are avoided thanks to safety limitations; $I_{\max }=915 \mathrm{~A}$ and $V_{\max }=30 \mathrm{kV}$ corresponding to electric field below $2 \mathrm{kV} / \mathrm{mm}$. Note that all the power injected into the plasma is not necessarily absorbed in the core, such that it is important not to confuse wave coupling with wave's absorption that will be discussed in the next subsection. To propagate, ICRF fast waves need a minimal density $\left(\sim 5.10^{18} \mathrm{~m}^{-3}\right.$ for dipole phasing excitation at $55.5 \mathrm{MHz}$ as shown in Fig. $\left.4 \mathrm{~b}\right)$ that generally sits few centimetres in front of the antennas. Waves must therefore tunnel through an evanescence layer, and this section aims to explain how this layer can be shortened to improve the RF coupling. 
The first way is to reduce the radial distance between the antenna front face (or leading edge of the closest limiter to the separatrix $R_{\text {antenna }}$ ) and the separatrix $\left(R_{\text {ext }}\right)$. This distance is often also called the radial outer gap ( $R O G=R_{\text {antenna }}-R_{\text {ext }}$ ) as in Fig.11 and graph e of Fig.A1 in Appendix, assessed from the magnetic reconstruction with NICE code $[46,47]$. However, this method is limited by a trade-off between coupling efficiency and heat loads strongly linked with impurity production.

The second most efficient way to improve antenna loading is of course to increase the plasma density [45]. As a result, the density in the edge plasma increases as well, shortening the evanescence layer. Limitations also exist since working at high densities (close to Greenwald limit) tends to trigger magnetohydrodynamic instabilities and leads to disruption.

The third most efficient way is to increase wave frequency $[45,48]$. The resonance should yet remain as close as possible to the central plasma and to the region where ions are well confined to minimize ripple losses and optimize fast ions thermalization.

Another way to improve ICRF loading is to fuel the plasma using gas valves in the vicinity of ICRF antennas, to locally increase the SOL density without impacting too much the central plasma density, tricky with low pumping efficiency. This method which proved good results in JET [24] and ASDEX Upgrade [49], is beyond the scope of the present study but more details can be found under [50].

Finally, LH power was also found to significantly help increasing density in the SOL more than in the core (Fig.7) which was defined as a figure of merit for improving the coupling efficiency of ICRF waves without losing control of the central density as shown in [50]. As more power is injected, it is known that more goes in the SOL and contributes to ionizing neutrals and increasing the SOL electrons density without changing the electron temperature [37]. However, other synergistic mechanisms may also contribute to the beneficial effect of LHCD on the ICRF coupling observed experimentally. A similar observation was also made in EAST [25], but so far, more experiments are needed to fully identify the dominant underlying mechanism.
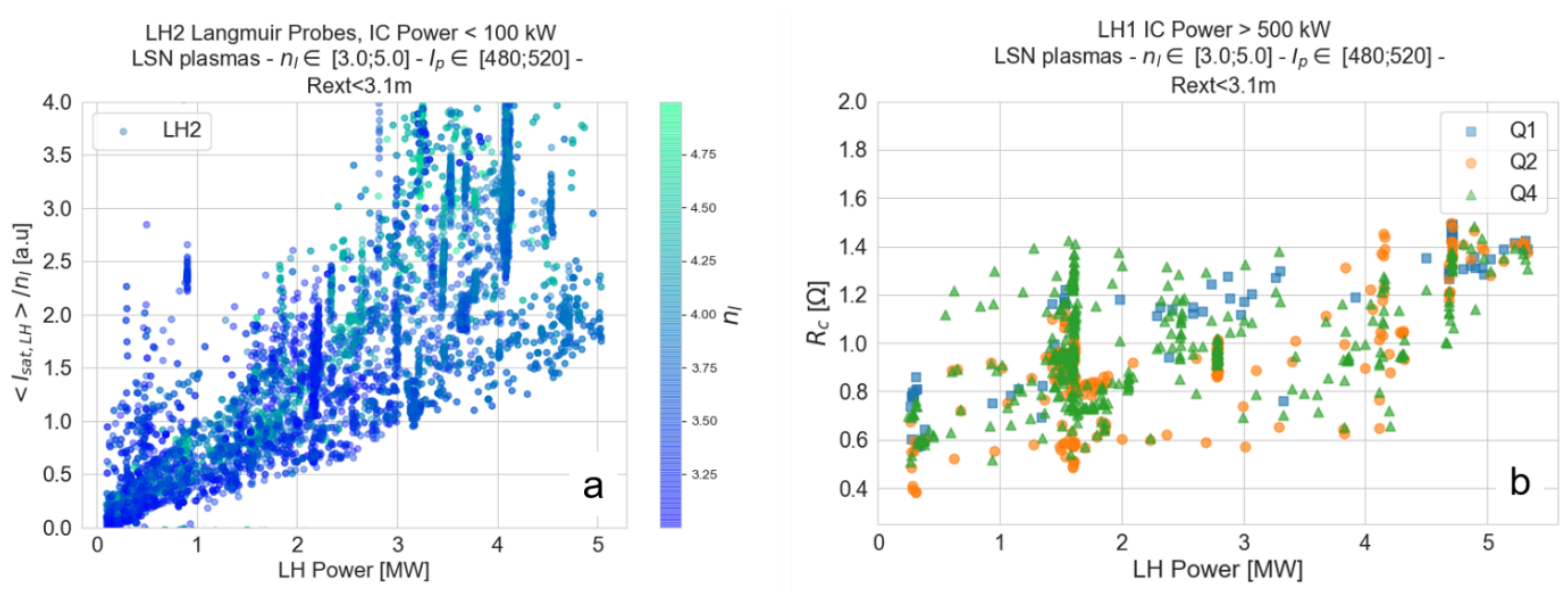

FIG. 7. (a) Ratio of ion saturation current measured by Langmuir probes fixed on LH2 launchers corners (a proxy for SOL density) over the line integrated density $\left(n_{1}\right)$ from interferometry (proportional to core density) as a function of LH power. Only discharges from 2018 and 2019 with low ICRF power $P_{\text {ICRF }}<100 \mathrm{~kW}, 3<\mathrm{n}_{1}<5.10^{19} \mathrm{~m}^{-2}, 480<1 \mathrm{p}<520 \mathrm{kA}$ and $\mathrm{R}_{\text {ext }}<3.1 \mathrm{~m}$ were selected and (b) ICRF antennas average coupling resistances as a function of the LH power. 


\subsection{ICRF wave absorption}

The ICRF heating efficiency is defined as the ratio between the power absorbed in the bulk plasma and the RF power launched by the antennas. This efficiency is related to the single-pass absorption (SPA) of the injected RF waves which depends, among other things, on the concentration of the resonant minority species. According to calculations done with the TOMCAT code [51] for the parameters used in these experiments, the hydrogen concentration should be around $8 \%$ to maximize the single-pass wave absorption (Fig.8). To experimentally benchmark this prediction, a scan of the hydrogen concentration was performed over a few discharges. In our study, the isotopic ratio $(H / H+D)$ is assessed by visible spectroscopy with high spectral resolution $(0.005 \mathrm{~nm})$ in order to capture the Zeeman effects on $\mathrm{H} \alpha(6562.1 \mathrm{~nm})$ and $\mathrm{D} \alpha(656.1 \mathrm{~nm})$ lines [52]. Measurements are performed with a time resolution of $0.5 \mathrm{~s}$.

For each discharge, a break-in-slope (BIS) analysis [53] of the plasma temperature response to the RF power switch-off was performed. This method assumes that the plasma temperature responds linearly to an RF power step if a short enough time interval is considered; $\Delta \mathrm{t}^{\sim 30 \mathrm{~ms}}<$ confinement time $\in[50,300 \mathrm{~ms}]$, (cf. Fig.5f \& Fig.9). The typical time scale of radiation increase due to ICRF power step is rather in the range of $200 \mathrm{~ms}$ (cf. Fig.6f around 4.6s, just after the period materialized by "BIS") so that it can be neglected over a short period $(50 \mathrm{~ms})$. The heating efficiency is $d / d t\left(3 / 2\right.$. ne.kb. $\left.\mathrm{T}_{i}\right)$, but since Ti is not measured in WEST, we will assume $T_{e}=T_{i}$ and assess $d / d t$ (3/2.ne.kb. $\left.T_{e}\right)$ for each ECE line instead. Note this is an underestimation since a larger fraction of the power is deposited on the hydrogen ions as shown in Fig. 8 and the difference between the neutrons signal and the electron temperature in Fig.5. The electron temperature response of all the ECE channels was computed and the results were integrated over the whole plasma to determine the absolute fraction of the injected power absorbed by electrons. If we assume that the electrons and ions pressures are the same, such that both species absorb the same fraction of RF power, we can estimate the total RF heating efficiency $\eta$ tot as:

$$
\eta_{t o t}=\left[2 \times \int \frac{d}{d t}\left(\frac{3}{2} n_{e} k_{B} T_{e}\right) d V\right] / \Delta P_{e f f}
$$

where the factor 2 accounts for electrons and ions and $\Delta P_{\text {eff }}=\Delta P_{I C R F}-\Delta P_{\text {rad }}$ the effective input power change during the RF power step, taking into account the fast changes in the radiation observed in the experiments (see Fig.9). This quantity gives, in absolute terms, an estimation of the fraction of the RF power absorbed in the plasma and includes, a priori, fast time-scale effects such as fast ion losses. The RF heating efficiency as expressed in eq. 4 corresponds to the red triangles in Fig.8.

Note first that TOMCAT predictions and the experimental results from break-in-slope (BIS) agree very well qualitatively, both showing optimal absorption conditions for intermediate $\mathrm{H}$ concentrations (4-8\%). Note that applying BIS analysis on a single central chord of the ECE (blue dots) already allowed capturing the right trend. As per quantitative estimations, we see that the heating efficiency is higher than the calculated SPA at low concentrations and lower near the predicted peak absorption.

As mentioned before, this is related to the facts that when the SPA is low $(<50 \%)$;

(i) the waves may perform several passes through the plasma before all the power is absorbed and (ii) edge losses that become significant are yet not accounted for in the simulations. The first effect (i) explains why the heating efficiency is larger than the SPA at low $\mathrm{H}$ concentrations while the latter (ii) explains why it never reaches more than $80 \%$ even in optimal conditions. Thus, fast ion losses may also reduce the RF heating efficiency of a given scenario, but quantifying this effect is difficult, since other loss mechanisms (such as the edge losses) are also at stake. Note that this method will also be harder to apply to the edge ECE channels in presence of LH power whose local deposition can lead to perturbations of the ECE signals due to fast electrons.

In another session with a background of LH power, the hydrogen resonance was moved at three different radial locations by changing the toroidal magnetic field strength without changing the plasma 
position (Fig.10a). Raising the plasma current would then result in enlarging the region of good confinement defined as the part of the plasma where there is no magnetic mirror capable of trapping particles between two toroidal coils (where there is not trapping well seen by the particles). In these different cases, BIS was this time applied to $W_{\text {dia }}$ and results summarized in Fig.10b. Best heating efficiency was achieved as the resonance was the closest to the central region where the density is maximal (about $3 \mathrm{~cm}$ toward the low field side). As the resonance was shifted further away from the central region towards the high field side, the heating efficiency decreased down to about $40 \%$. This result suggests that on-axis heating is the best option, while no clear correlation with the plasma current was observed.

Despite these results confirm efficient ICRF heating in the plasma core if the $\mathrm{H}$ concentration is smaller than $10 \%$ and the resonance at the centre of the plasma, it is important to mention that the heating effect is often only transient and rapidly compensated by an increase in radiation. Due to RF sheath excitation, plasma surface interactions intensify immediately when ICRF is powered [50], but impurities take about $200 \mathrm{~ms}$ to reach the core (4 times longer than the time interval used for the BIS method). Depending on the plasma and in particular, on the wall conditions (boronization being helpful to reduce the RF-induced impurity influx $[33,54])$, the central temperatures may drop back to the original values before the application of ICRF (all the RF power being effectively transformed into radiation) or, in extreme cases, even lead to plasma cooling [55], as shown in Fig.5.

Assuming that methods to maximize the wave-plasma coupling were fairly understood and that we have evidence of efficient wave absorption in the core, further optimization must come from a better understanding of the mechanisms leading to core contamination by RF-induced plasma-wall interactions and potentially mitigating actuators, to which the last section is devoted.

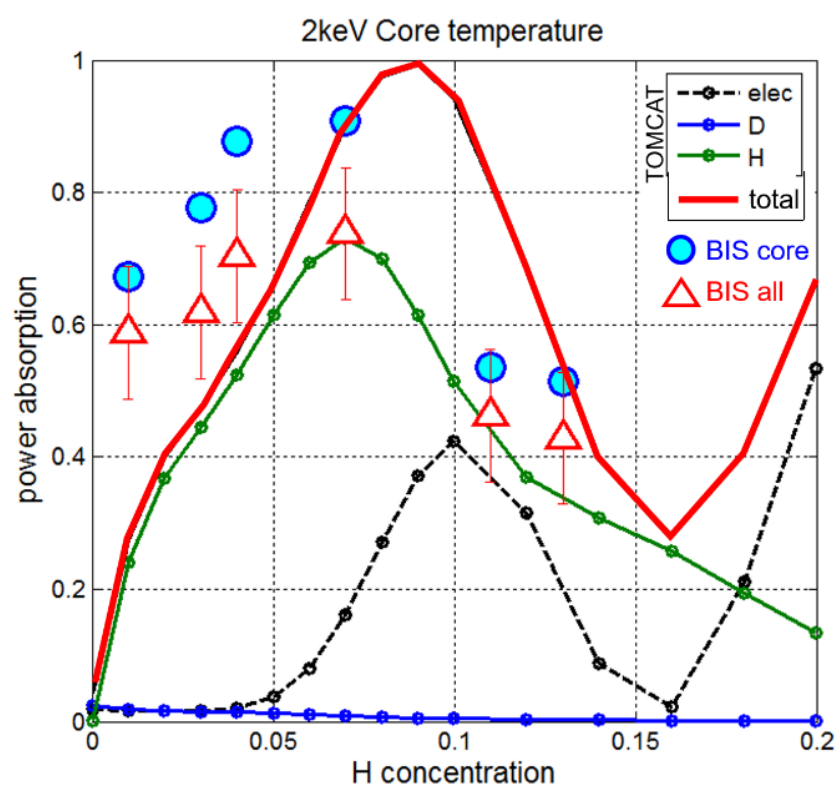

FIG 8. Predicted single-pass absorption (computed with the TOMCAT code) and RF heating efficiency (inferred from the break-in-slope (BIS) method on the ECE response due to RF power steps cf. Fig. 10) as a function of the $\mathrm{H}$ concentration for a series of similar discharges (53700-05) 

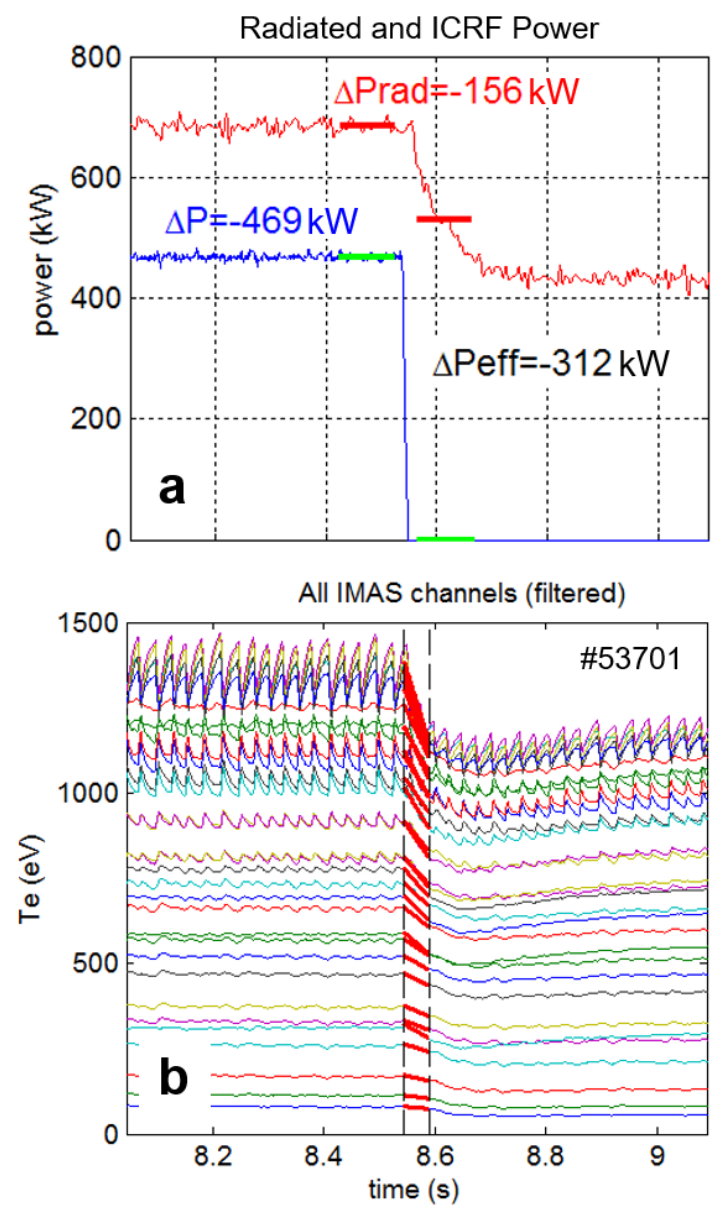

FIG 9. Time traces used for applying BIS analysis (break-in-slope) at the end of an ICRF pulse of (a) Radiated and injected ICRF power and

(b) all ECE channels representing electron temperature at the different radius.
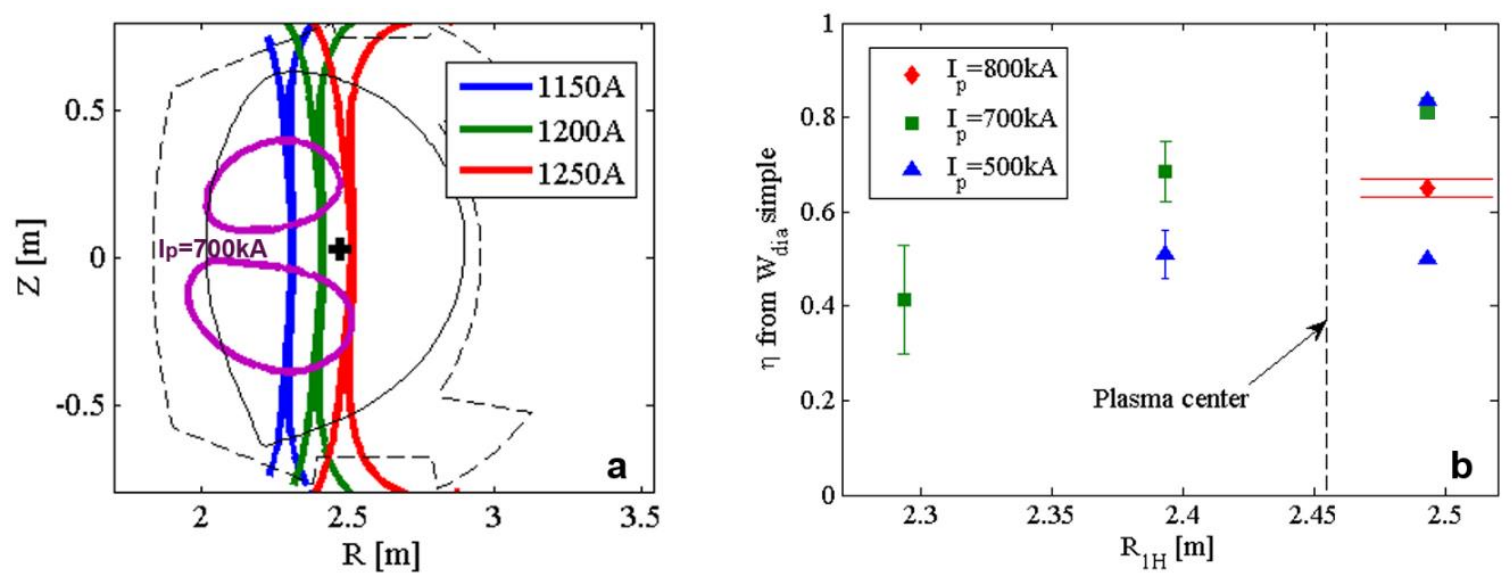

FIG 10. (a) Displayed on a poloidal cut are the respective positions of hydrogen resonance for three different poloidal currents, and the region of good confinement enlightened in purple for a plasma with a current of 700kA and (b) The evolution of the absorption efficiency assessed with BIS analysis on $\mathrm{W}_{\text {dia }}$ for different plasma currents and radial positions of the hydrogen resonance. 


\section{Plasma surface interactions}

\subsection{Position of the separatrix}

The last closed flux surface of a tokamak plasma is commonly called the separatrix as it separates the confined plasma with closed magnetic field lines from the SOL with open field lines (cf. red surface in Fig.A2). In the SOL plasma, temperature and density drop exponentially with short widths. In nominal WEST plasma scenarios, the first objects (limiters) are sitting few centimetres away from the separatrix. All parameters equal elsewhere, as the plasma is moved away from the antennas (ROG increase), one would expect temperature and density to drop at the limiter, resulting in a decrease of these sources. In addition, moving the plasma away from the limiter sources may also lower the core contamination from these sources, acting on the screening and transport of impurities.

A database containing more than 300 discharges, was built by averaging many signals over $0.5 \mathrm{~s}$ plateaus during which the plasma current and total power changed less than $5 \%$. Since ICRF pulses are more rarely fulfilling this criterion than LH pulses, ICRF-related phenomena are underrepresented. Relying on the database, Fig.11 shows that increasing the ROG results in a drop in the fraction of radiated power $\mathrm{f}_{\mathrm{rad}}=\mathrm{P}_{\mathrm{rad}} / \mathrm{P}_{\text {tot }}$.

One should yet remain cautious for RF-heated discharges, since they can also be subject to tradeoff between the interaction of their limiters with the plasma and their coupling efficiency (as stressed in section 3.2). For example, when moving the plasma radially away from the antennas, one should not exclude the possibility that the gain on impurity production would be compensated by an increase of the electric field on the antenna structure due to less efficient coupling. An optimal must be found between the amount of power that can be coupled to the plasma and the fraction of it that is being radiated. This will be discussed in the next parts.

Based on the colors in Fig.11, note also see that the power crossing the separatrix $\left(P_{\text {sep }}\right)$ seems rather independent of the fraction of power radiated nor the ROG.

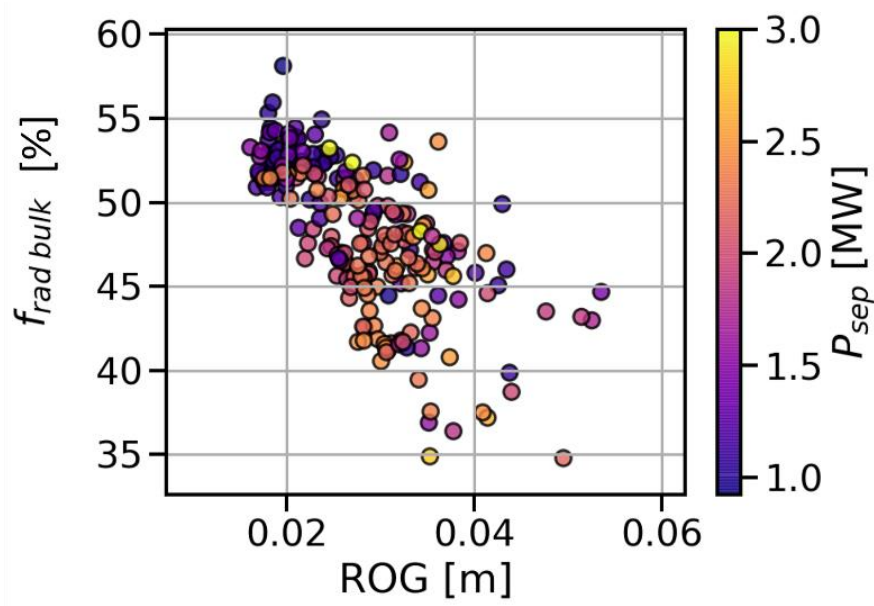

FIG 11. Fraction of power radiated $\left(f_{\text {rad bulk }}=P_{\text {rad bulk }} / P_{\text {injected }}\right)$ as a function of the radial distance between the antenna limiter edge $\left(R_{\text {antenna }}\right)$ and the separatrix $\left(R_{\text {ext }}\right),\left(R O G=R_{\text {antenna }}-R_{\text {ext }}\right)$. Points colours are a function of the power crossing the separatrix $\left(P_{\text {sep }}=P_{\text {injected }}-P_{\text {rad }}\right)$. Only were taken into account discharges with $3<n_{1}<5.10^{19} \mathrm{~m}^{-2}, 480<\mid \mathrm{p}<520 \mathrm{kA}, \mathrm{P}_{\text {aux }}>1.5 \mathrm{MW}, \mathrm{f}_{\mathrm{rad}}>20 \%$ and Tecenter $>2 \mathrm{keV}$.

\subsection{LH power alone}

In the next sections, we present observations of RF power influence on impurity sources. We will extensively use visible spectroscopy data as detailed in section 1.2. Figures $12-15$ represent for various 
cases, respectively (a) the deuterium and (b) tungsten fluxes estimated from $\mathrm{DI}_{\delta}(\delta$ line of the Balmer series of neutral deuterium) and WI (neutral tungsten) calibrated data $\left(\mathrm{Ph} / \mathrm{m}^{2} / \mathrm{s} / \mathrm{sr}\right)$ and $(\mathrm{c})$ their ratio as a proxy of the effective sputtering yield (cf. eq.3) along Q2 antenna right limiter (Fig.12-15). In green is always represented the level in ohmic cases so to visualize the increase induced by various heating systems. In the first case (Fig.12), we show the influence of 5MW LH power along discharge 55802 detailed in the appendix. Rather consistent with the fact that $\mathrm{LH}$ power leads to an increase of the electron density in the edge discussed earlier (Fig.6), the consequence on the deuterium influx is visible on the graph to the left. The effective sputtering yield also increases in a fairly homogeneous fashion along with the poloidal height, which could either be the result of an

(i) increase of electron temperature $\left(T_{e}\right)$

(ii) increase of ions temperature $\left(T_{i}\right)$

(iii) increase of ionization states $\left(Z_{i}\right)$

(iv) change in plasma composition $\left(Z_{\text {eff }}\right)$

As mentioned in section 1.2, shown in Fig.12 and [37], (i) is not expected to play a major role since $\mathrm{T}_{\mathrm{e}}$ at the target is rather constant (between 15 and $20 \mathrm{eV}$ ) according to Langmuir probes, except at the divertor target where it can drop below $5 \mathrm{eV}$ in case of detachment. $\mathrm{T}_{i}$ can grow either relatively to $T_{e}$ due to specific radiation losses to electrons, or absolutely if part of the LH power is deposited onto ions, increasing their sputtering yield. This could for instance be the result of mode conversion or parametric decay making possible direct wave power deposition on the ions [56]. Or also, the screening effect of fast-electrons by high- $Z$ impurity could be another explanation being investigated $[57,58]$. In the case of tungsten, this effect is dominant on low ionization states $(Z<20)$ therefore in the edge of the plasma where no wave absorption occurs. Fast electrons would then lose energy on these high- $Z$ impurity ions, either raising their temperature (ii) but mostly their state of ionization (iii). These effects will be further studied, both experimentally using Retarding Field Analyzer to measure $T_{i}[37,59]$, and hopefully benchmarked by modelling with LUKE code [60] into which the dynamics of fast electrons in presence of partially ionized high-Z atoms was recently implemented. Finally (iv), it is also likely that powering LH changes the composition of the plasma by injecting some impurities such as copper from the launchers which typically increase as seen on VUV spectral line. Note the position of the peak is consistent with the larger poloidal curvature of the plasma compared to the antenna radius, leading to a smaller ROG at the centre than at the corners.
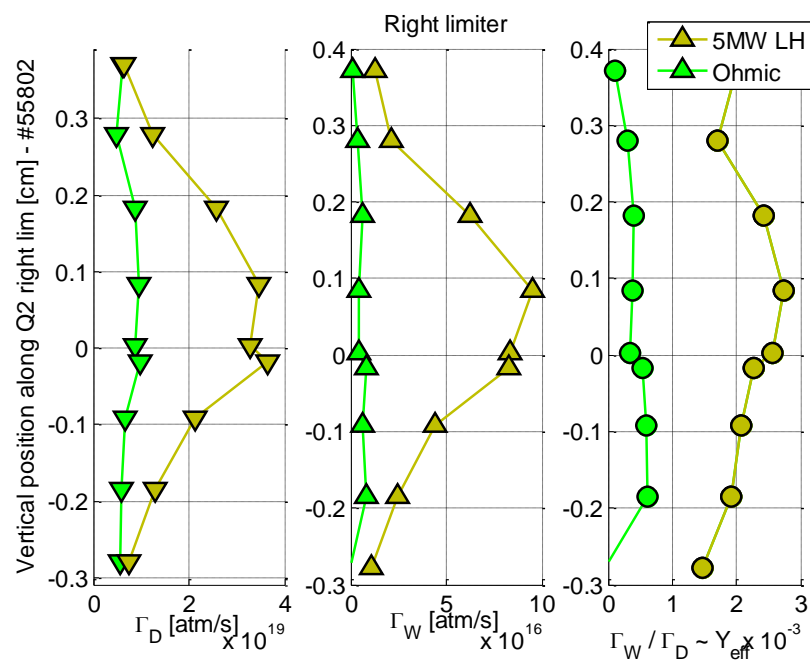

FIG 12: Deuterium and tungsten fluxes estimated from $\mathrm{DI}_{\delta}$ and $\mathrm{WI}$ calibrated data $\left(\mathrm{Ph} / \mathrm{m}^{2} / \mathrm{s} / \mathrm{sr}\right)$ poloidal profiles along Q2 antenna right limiter with $S / X B$ coefficients determined in section 1.2. Green profile stands for ohmic case while yellowish case for a discharge heated with $5 \mathrm{MW}$ LH power. 
In another experiment, three different levels of LH power were performed from 1 to $2 \mathrm{MW}$. Averaged profiles of particle fluxes along Q2 right limiter are represented by the grey curves in Fig.13. In this rather small power range, the change in limiter sources - yet quite small - was fairly consistent with the power increase, while no significant variation was observed on the divertor. As the LH power increased, the deuterium flux increased consistently. Despite slight increase of the electron temperature registered by reciprocating probes on the APL magnetically connected to the limiter (cf. blue line in Fig.1), the tungsten sources changed little along the power scan [61]. Grey profiles in Fig.13 give an idea of sources level along this experiment [61]. This suggests that the deuterium influx is rather correlated to the LH power. This is consistent with the fact that electron temperature and density both increase together with the lower hybrid power. Yet, sputtering yields likely remaining in a region of small values or maybe even under threshold values in most regions, raise in deuterium flux did not cause a significant increase of the tungsten sources.

To compare, the next section will now present similar discharges, only heated with ICRF.

\subsection{ICRF power}

When powering the three ICRF antennas with 500kW each, on the red profiles in Fig.13, we first see that the deuterium influx is similar than in ohmic case, consistent with the fact that the deuterium influx is rather correlated to the LH power as explained in the previous section. With ICRF however, a large increase of the effective sputtering yield - resulting from RF sheath excitation on antennas limiters - leads to an increase of W sources larger than when the same amount of power is launched with $\mathrm{LH}$. As explained in the previous subsection, mechanisms by which LH enhances plasma surface interaction are roughly linear, and result mostly from global interactions between electrons and ions, a combination of linear thermal sheaths and turbulent process [62]. On the contrary, RF sheath excited by ICRF are highly non-linear [3] and dominantly local (despite broadening of interactions in case of low single-pass absorption $[9,50]$ which was a priori not the case during these discharges). This can for instance be illustrated by switching off two ICRF antennas and adding 500kW on Q2, this way the total ICRF power decreases from 1.5MW down to $1 \mathrm{MW}$ on a single antenna. As shown in the blue profiles in Fig.13a, the deuterium flux does not change. The effective sputtering yield and $\mathrm{W}$ sources however increase further on Q2 limiter, clearly showing the character local and deleterious of ICRFinduced interactions through RF-sheath excitation on close and magnetically connected PFCs. Notice in all these cases, LH and ICRF were so far studied separately to help to identify their respective role on each parameter, which leads to the conclusion that LH mostly acts on the deuterium influx whereas ICRF mostly acts on the sputtering yield. The last section finally presents what happens when both are powered together.

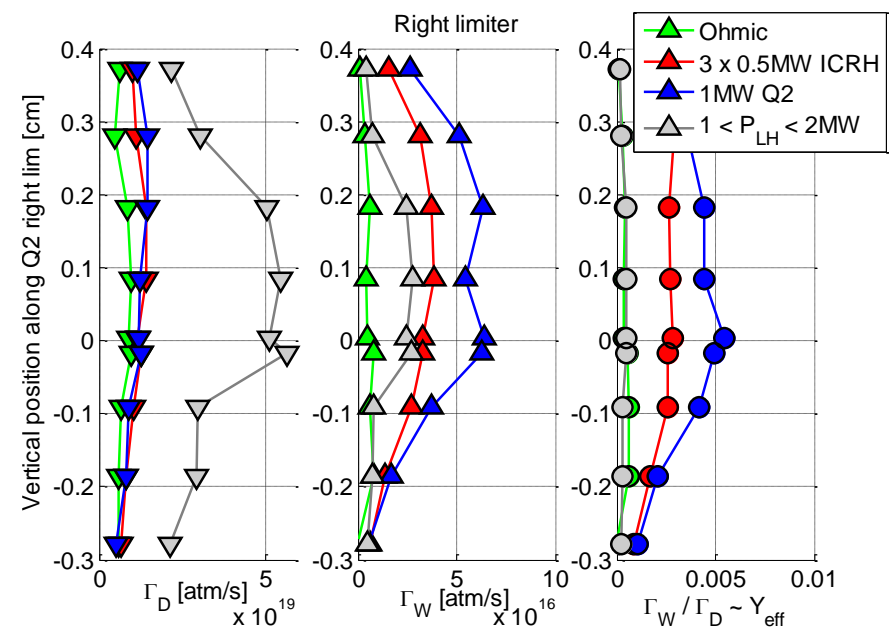

FIG 13: Deuterium and tungsten fluxes estimated from $\mathrm{DI}_{\delta}$ and WI calibrated data $\left(\mathrm{Ph} / \mathrm{m}^{2} / \mathrm{s} / \mathrm{sr}\right)$ poloidal profiles along Q2 antenna right limiter with $S / X B$ coefficients determined in section 1.2. Different profiles are superposed for different heating schemes. 


\subsection{High power discharges with LH and ICRF}

With previous approaches, we have shown that LH essentially acts on the deuterium influx with little influence on the effective sputtering yield compared to ICRF. We therefore expect the synergy of both heating systems to enhance impurity production even more. In this last section, we focus on a highpower discharge, particularly relevant for regimes foreseen on future devices like ITER and CFETR. As can be seen in Fig.14, powering ICRF resulted in a very strong increase of the sputtering yield peaking at the equatorial plane. Combined with an additional increase of the deuterium flux presumably due to LH (cf. section 4.2), the consequence is a serious increase of W sources along the limiter, also peaking at the midplane (consistent with the larger curvature of the plasma with respect to the antenna limiters). This could be related to excitation of RF-sheath at the centre stronger than usual, for instance, due to the large septum, which should be addressed in a dedicated study with modelling tools such as RAPLICASOL [63] and/or TOPICA [64-66] and SSWICH codes [40].

In comparison, we also monitored similar quantities along the divertor (Fig.15) and found peaks at the inner and outer targets with similar order of magnitudes to these measured on limiters. Nevertheless, unlike limiter sources, divertor sources remain exactly the same with and without ICRF. This is because the power balance is globally unchanged during ICRF application since radiation compensates heating. As a consequence, the sputtering on the divertor remains constant as well as the sources.

We can now estimate the intensity of the main impurity sources. If we consider that $W$ sources in the divertor are homogenous toroidally and the sum of the inner $\left(2.07<R_{\text {in }}<2.12 \mathrm{~m}\right)$ and outer $\left(2.25 \mathrm{~m}<\mathrm{R}_{\text {out }}<2.3 \mathrm{~m}\right)$ strike points regions (giving a wetted surface of $\left.S_{\text {divertor }}^{\text {wett }}=1.4 \mathrm{~m}^{2}\right)$ with average W fluxes of the order of $\Gamma \mathrm{w}=10^{18}$ ions/s. We can estimate the $\mathrm{W}$ sources in the divertor (as a number of tungsten ions released per second) when active ICRF antennas are magnetically connected:

$$
\Gamma_{W \text { divertor }}^{\text {total }} \approx \Gamma_{W \text { divertor }}^{\text {average }} \times S_{\text {divertor }}^{\text {wetted }}=1.4 .10^{18} \text { ions } / s
$$

As far as sources on limiters are concerned, we can look at the right limiter of Q2 antenna and estimate the same quantities to assess the intensity of each source. For the ten antenna limiters of the chamber (APL sources usually ten times smaller as it is retracted during the shots $\sim 2 \mathrm{~cm}$ behind antenna limiters), we can take $\Gamma_{\mathrm{w}}=4.5 .10^{17}$ ions $/ \mathrm{s}$ over $S_{\text {limiter }}^{\text {wetted }}=10 \mathrm{~cm} \times 50 \mathrm{~cm}=0.05 \mathrm{~m}^{2}$ toroidal-poloidal range roughly around mid-plane location, we can get an estimation of $\mathrm{W}$ sources from a limiter magnetically connected to an active ICRF antenna:

$$
\Gamma_{W \text { limiter }} \approx \Gamma_{W \text { limiter }}^{\text {average }} \times S_{\text {limiters }}^{\text {wetted }}=2.25 .10^{16} \text { ions } / s
$$

In the worst case scenario, with all ICRF antennas powered and RF-sheath induced on all antenna limiters, we find with these rough estimations that sources from 5 antennas or 10 limiters would amount to a sixth of divertor ones.

Even if the erosion is generally weaker on limiters than in divertor targets, impurities are in principle better screened and promptly redeposited when emitted from the divertor - due to an increase of collisionality - whereas they more easily penetrate in the core when emitted from antenna limiters - turbulent transport with ware effect - as seen in Alcator C-Mod [4, 67] and ASDEX Upgrade [8]. In addition, spectroscopic measurements in the divertor regions are made at the maximum of ripple modulations, which could lead to an overestimation of divertor sources. For example, even if analogies between erosion and heat loads phenomena are not straightforward [68], the toroidal average of divertor heat loads in WEST is about a factor 2 lower than the peak. For sources of a similar order of magnitude, limiters are therefore expected to account for a large amount of $\mathrm{W}$ in the plasma.

Note this is also consistent with the preliminary observation while scanning the phase of the Q1 antenna (Fig.5). Rigorously assessing penetration factors would be the best way to answer quantitatively to this question, but without calibrated VUV spectrometer, assessing a proxy is so far only feasible very indirectly, making assumptions on the main radiating species and comparing 
radiations from bolometry with synthetic diagnostics fed with profiles of electron temperature and density mapped with equilibrium reconstruction. Still, in this discharge - and most of others at highpower -, since powering ICRF results in (i) a relative increase of impurity lines brightness measured by VUV spectrometer (cf. graph c in Fig.A1 in Appendix), (ii) a global temperature drop resulting from impurity radiation (cf. graph d in Fig.A1), (iii) an increase of W sources on limiters (Fig.14) and (iv) no change of W sources in the divertor (Fig.15), we suspect antennas limiters to be the sources dominantly responsible for core contamination by high-Z impurity. Notice the erosion of silver from Q4 antenna Faraday Screen also contributes in cooling the plasma even more and increase the radiated power (cf. graph b, $c$ and d in Fig.A1).

Finally, we show the evolution of the tungsten source on an ICRF antenna limiter (Fig.16a) and on the outer target of the divertor (Fig.16b) as a function of the total radiated power. In "low" power discharges $\left(P_{\text {tot }}<5 \mathrm{MW}\right)$, we see that the radiated power is well correlated to both sources. However, in "high" power discharges $\left(P_{\text {tot }}>5 \mathrm{MW}\right)$, the total radiated power appears well correlated to the tungsten sources from active antenna limiters, but less correlated to the tungsten sources from the divertor outer target, which slope flattens a bit (cf. graphs b \& d). When an ICRF antenna is not powered through, note its limiters seem to have negligible influence on the power radiated, as shown by the group of points in Fig.16a with "Q2 inactive". This also suggests that for discharges at high RF power, tungsten sources in active ICRF antennas limiters can have a greater impact on the radiated power than sources of the divertor.

At this point it is worth mentioning that experimental results have also been benchmarked by modelling with Soledge2D-EIRENE [69] and ERO2.0 [70], benchmarking that for WEST plasmas, divertor screening is very efficient in these scenarios, in particular impurities emitted from the outer target, while these emitted from the inner target flow to the high field side without penetrating much inside the separatrix. However, the situation can change quite a lot for divertor sources depending on divertor state; $\mathrm{W}$ sources at a given divertor leg typically drops by an order of magnitude when it detaches, in addition to changes of impurity transport which won't be discussed.
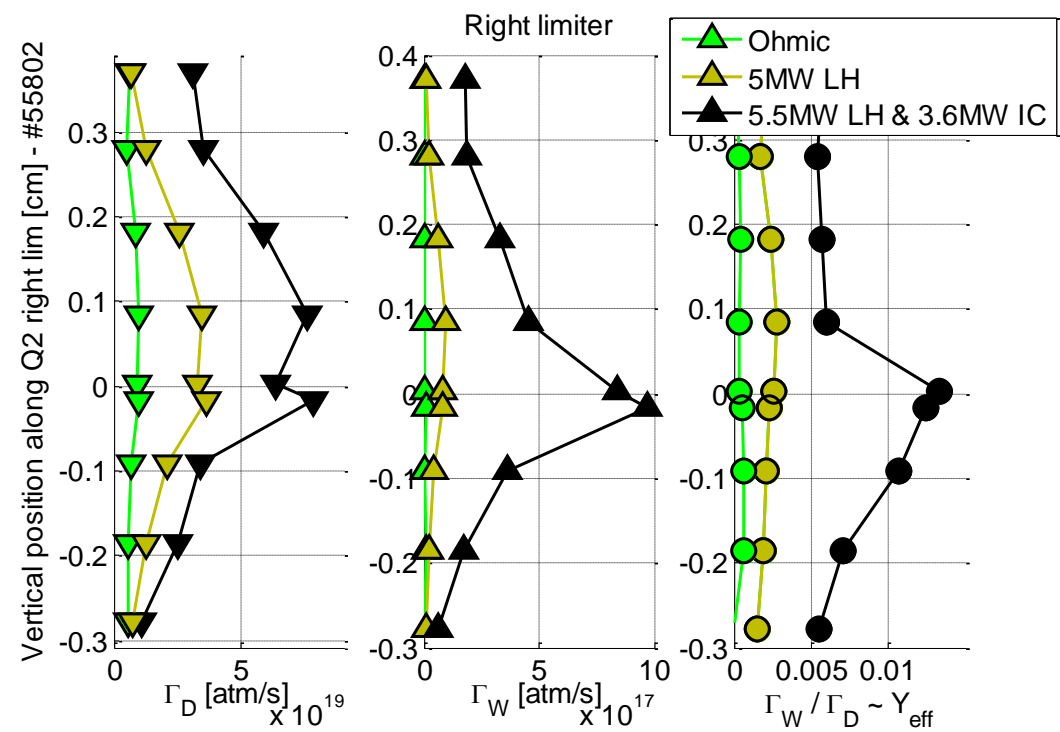

FIG 14: Deuterium and tungsten fluxes estimated from $\mathrm{DI}_{\delta}$ and $\mathrm{WI}$ calibrated data $\left(\mathrm{Ph} / \mathrm{m}^{2} / \mathrm{s} / \mathrm{sr}\right)$ poloidal profiles along Q2 antenna right limiter with S/XB coefficients determined in section 1.2. Green and yellow profiles are the same as in Fig.12, and black profiles correspond to the last part of the same discharge with 5.5MW LH and 3.6MW ICRF power, characteristic of WEST highpower L-mode discharges. 

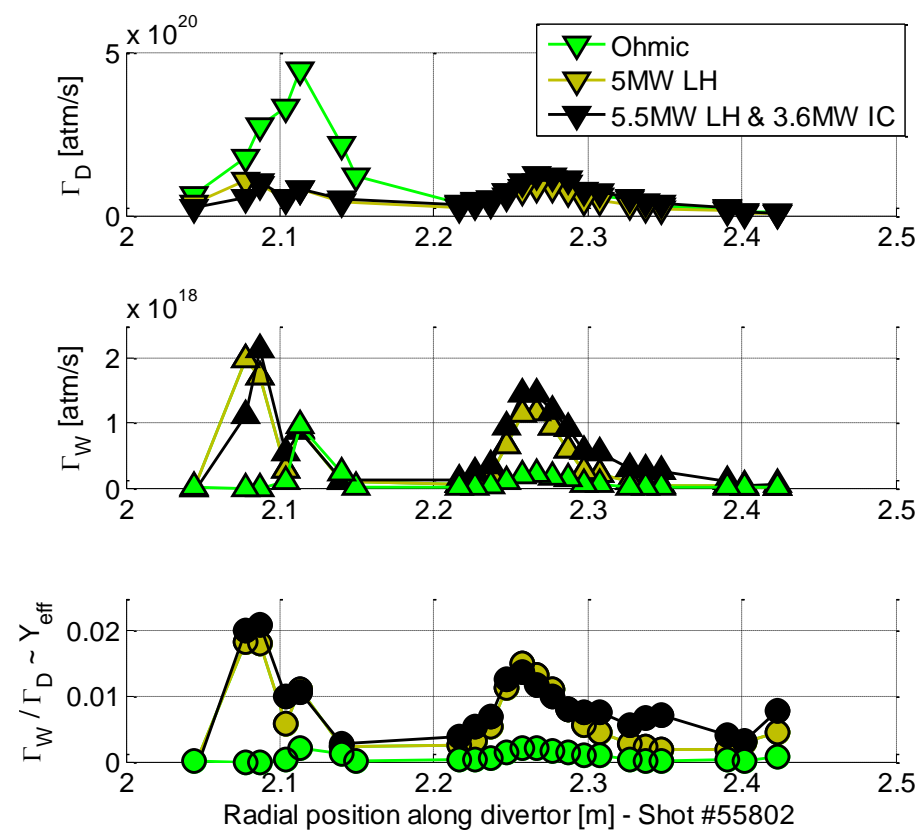

FIG 15: Deuterium and tungsten fluxes estimated from $\mathrm{DI}_{\varepsilon}$ and WI calibrated data $\left(\mathrm{Ph} / \mathrm{m}^{2} / \mathrm{s} / \mathrm{sr}\right)$ radial profiles along divertor targets with $S / X B$ coefficients determined in section 1.2. Same cases as in Fig.12.
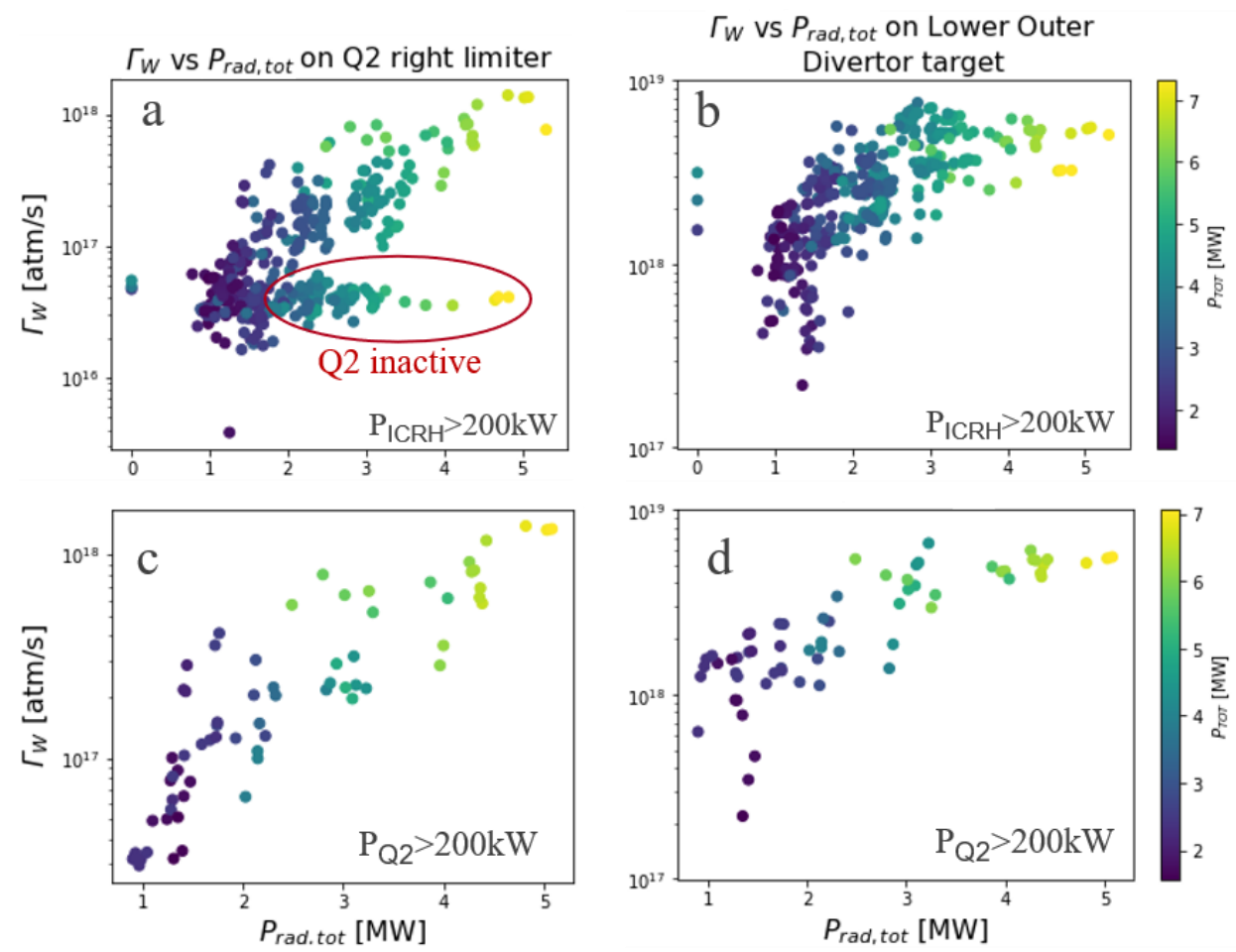

FIG 16: Evolution of the tungsten sources on an ICRF antenna limiter (a \& c) and on the outer target of the divertor ( $b \& d$ ) as functions of the total radiated power, and coloured with the total power injected. Top graphs ( $\mathrm{a} \& \mathrm{~b}$ ) represent all points with ICRF regardless of which antenna was powered, while bottom graphs (c \& d) only show cases with $Q 2$ antenna powered.

Tungsten fluxes are estimated from WI calibrated data $\left(\mathrm{Ph} / \mathrm{m}^{2} / \mathrm{s} / \mathrm{sr}\right)$ with local S/XB coefficients. These statistic results rely on the same database as the one used in section 4.1 to build Fig.11. 


\section{Conclusions and perspectives}

This study aims at reporting observations regarding plasma surface interactions on RF-heated WEST L-mode discharges. For this matter, it first focusses on a key prerequisite for the study, namely the optimization of the ICRF-system.

The first aspect to optimize is the wave coupling by finding best compromizes between various parameters: (i) minimize the distance between antenna and plasma; to the cost of stronger interactions and heat loads (ii) globally increase plasma density (iii) increase wave frequency (iv) increase lower hybrid power; helps increasing density at the edge more than in the core and (v) locally increase plasma density by injecting gas through valves located near antennas. The next aspect to optimize is the wave absorption in the minority heating scheme. This is achieved by preserving the right concentration of hydrogen (between 6 and 10\%) and placing the ICRF resonance at the centre.

Once such appropriate conditions fulfilled, impurity sources are investigated in different scenarios. The complexity of the cases studied increases gradually, starting with plasmas only heated with LH, then plasmas only heated by ICRF, in order to methodically disentangle key effects proper to each heating system. Finally, discharges combining both heating systems at high power levels are studied, providing a more holistic understanding of the topic.

Focusing first on discharges only heated with lower hybrid, increasing the LH power lead to an increase of the deuterium influx on the limiters. This is rather consistent with an increase of SOL density, beneficial for the ICRF coupling. While these effects are rather global, the effective sputtering yield only seems to increase in a moderated and linear fashion with $\mathrm{LH}$.

On the contrary, focusing next on WEST L-mode discharges only heated with ICRF, while ICRF has little influence on the deuterium influx, it can have substantial effects on the effective sputtering yield, via the excitation of the so-called RF sheath, as seen in other machines [13]. As long as parameters are well-tuned for optimizing wave absorption, these effects remain localized in regions close and magnetically connected to the active antennas (near fields). Otherwise, they can spread to all regions (far fields) [50]. Hence the importance to achieve accurate control of the minority concentration.

Finally, combining LH with ICRF, implies dealing with the synergy of their respective deleterious effects. Therefore, the consequence of the simultaneous increase of the deuterium influx and the effective sputtering yield, is a sharp increase of the tungsten sources.

As far as plasma contamination by impurity and radiation losses are concerned, the situation remains manageable at low power levels (total RF power under 5MW). However, due to the non-linear behaviours of both RF-sheath excitation and effective sputtering yield, increasing further the total RF power comes with great challenges. At high power levels (total RF power over 6MW), it is found that limiter sources can reach similar order of magnitude as divertor sources.

In addition, several complementary modelling studies were performed, combining SolEdge2DEIRENE [69] and ERO2.0 [70] codes. Note that these studies neglect ballooning transport in the edge and that the antenna limiter position was set $15 \mathrm{~cm}$ further from the plasma than in real conditions. Even under these assumptions was found that among key impurity sources (upper divertor, inner bumper, inner and outer divertor targets) antenna limiters seem to have the highest plasma contamination efficiency. Since the screening of impurity seems to be much better in the divertor region than nearby antenna limiters, we believe that in presence of ICRF, the antenna limiters could be the principal contributors to core contamination by tungsten.

As far as perspectives of optimization are concerned, the following upgrades could in theory be possible, but would require substantial engineering efforts :

1) Active limiters; insulated and biased to actively compensate RF currents

2) Use a tilted antenna to align it with the magnetic field lines (Fig.2b) and reduce the ratio of parasitically excited slow wave with respect to fast wave such as in Alcator C-Mod [71] 
3) Use another antenna with more than two toroidal-arrays of straps, to allow compensating image currents on limiters such as in ASDEX-Upgrade $[13,43]$

4) Change $W$ limiters into low-Z material (such as boron) [72]

5) Design and implement a travelling wave antenna [73]

- not only to reduce electric fields for a given coupled power

- but also to preserve relatively efficient loading by sitting of the order of $10 \mathrm{~cm}$ radially further in the SOL, where density and temperature are so low, that plasma surface interaction may drop down to marginal levels

So far, even if if the three first options are not envisaged, the two last upgrades (4 \& 5) are being seriously considered for WEST and further discussed under [74].

\section{Acknowledgments}

The whole WEST ICRF team, GMICS colleagues, and Olivier Meyer who used to develop the visible spectroscopy diagnostic in WEST, are all warmly acknowledged.

This work has been carried out within the framework of the French Federation for Magnetic Fusion Studies (FR-FCM) and EUROfusion Consortium and has received funding from the Euratom research and training program 2014-2018 and 2019-2020 under grant agreement No 633053. The views and opinions expressed herein do not necessarily reflect those of the European Commission.

This work was carried out under the framework of the Sino-French Fusion Energy centre (SIFFER, http://www.siffer.science/). 


\section{Bibliography}

[1] Bucalossi J. et al 2014 Fusion Eng. Des. 89 907-12

[2] R. Pitts, ITER full W divertor Final Design Review, June 2013

[3] L. Colas et al. Nucl. Fusion 43 (2003) 1-15

[4] B. Lipschultz et al Nucl. Fusion 41, No. 5 p.585 (2001)

[5] S.J. Wukitch et al. Journal of Nuclear Materials 363-365 (2007) 491-497

[6] S.J. Wukitch et al. Journal of Nuclear Materials 390-391 (2009) 951-954

[7] R. Dux et al. Journal of Nuclear Materials 363-365 (2007) 112-116

[8] R. Dux et al. Journal of Nuclear Materials 390-391 (2009) 858-863

[9] G. Urbanczyk et al 2020 Nucl. Fusion 60126003

[10] V. Bobkov et al. Journal of Nuclear Materials 438 (2013) S160-S165

[11] C. C. Klepper et al 2016 Phys. Scr. 2016014035

[12] A. Lasa et al 2018 Nucl. Fusion 58016046

[13] V. Bobkov et al. Nuclear Materials and Energy 18 (2019) 131-140

[14] N. Fedorczak et al. Journal of Nuclear Materials 463 (2015) 85-90

[15] M. Kubic et al. Journal of Nuclear Materials 438 (2013) S509-S512

[16] L. Colas et al. Plasma Phys. Control. Fusion 49 (2007) B35-B45

[17] L. Colas et al. EPS 2018 proceedings

[18] I. Cziegler et al 2012 Plasma Phys. Control. Fusion 54105019

[19] C. Gil et al. Fusion Eng. Design 2019,140 pp 81-91

[20] J.P. Gunn et al 2017 Nucl. Fusion 57046025

[21] A. Ekedahl et al. Proc. 21st Topical Conf. on RF Power in Plasmas, CA (2015)

[22] P Bibet et al. 1995 Nucl. Fusion 35 1213-23

[23] D Guilhem et al. Fusion Eng. Design 86 (2011) 279

[24] P. Jacquet et al. Nucl. Fusion 56 (2016) 046001

[25] G. Urbanczyk et al 2019 Nucl. Fusion 59066023

[26] J.L. Schwob et al. Review of Scientific Instruments 58, 1601 (1987)

[27] R. Guirlet et al 2019 JINST 14 C10036

[28] R. Dejarnac et al 2018 Nucl. Fusion 58066003

[29] Gunn J P and Pascal J-Y 2011 Rev. Sci. Instrum. 82123505

[30] J.P. Gunn et al Journal of Nuclear Materials 438 (2013) S184-S188

[31] A. Pospiesczcyk et al. J. Phys. B: At. Mol. Opt. Phys. 43 (2010) 144017

[32] O. Meyer et al. Rev. Sci. Instrum. 87, 11E309 (2016)

[33] Private communication with Nicolas Fedorczak on ICRF-induced sources in JET-ILW

[34] I. Beigman et al. Plasma Phys. Control. Fusion 49 (2007) 1833-1847

[35] Brezinsek et al. PSI conference 2012 (https://www-

amdis.iaea.org/meetings/AMPMI14/Presentations/AMPMI-2014-12-16-Talk-Brezinsek-Spectroscopy-

2by4.pdf)

[36] R. Dejarnac et al 2020 Fusion Eng. Des. 112120

[37] M. Kocan. "Ion temperature measurements in the scrape-off layer of the Tore Supra tokamak"

PhD 2009

[38] P.C. Stangeby 2000 The Plasma Boundary of Magnetic Fusion Devices - Institute of Physics Publishing Bristol and Philadelph (2000)

[39] V. Basiuk et al. Nucl. Fusion 44 (2004) 181-192

[40] J. Jacquot et al, Phys. Plasmas 21061509 (2014)

[41] Jean-Michel Bernard et al. Fusion Eng. Design 2017

(https://doi.org/10.1016/j.fusengdes.2017.05.024)

[42] W Helou et al. Fusion Eng. Design 96-97 (2015) 473

[43] L. Colas et al. Plasma Phys. Control. Fusion 59 (2017) 025014

[44] M Usoltceva et al 2019 Plasma Phys. Control. Fusion 61115011 
[45] J. Hillairet et al, (2021) Nucl. Fusion 104629

[46] Blaise Faugeras et al 2018 Nucl. Fusion 58106032

[47] J. Blum et al. 2012 Journal of computational physics, 231(3), pp.960-980

[48] W. Helou et al, AIP Conference Proceedings 2254, 030009 (2020)

[49] W. Zhang et al Nucl. Fusion 56 (2016) 036007

[50] G. Urbanczyk et al, AIP Conference Proceedings 2254, 030012 (2020)

[51] D. Van Eester and E. Lerche. Plasma Phys. Control. Fusion 55 (2013) 055008

[52] M. Koubiti et R.R. Sheeba. Atoms 2019, 7, 23

[53] E. Lerche et al. Plasma Phys. Control. Fusion 50 (2008) 035003

[54] V. Bobkov et al. 2013 Nucl. Fusion 53093018

[55] L. Colas et al. AIP Conference Proceedings 2254, 040004 (2020)

[56] V. V. D'yachenko et al. Plasma Physics Reports 45, (2019)

[57] Private communications with Yves Peysson

[58] K. Król et al. « Screening effect of partially ionized high-Z impurities in relativistic electron

Fokker-Planck calculations and runaway electron dynamics »

(https://rifj.ifj.edu.pl/bitstream/handle/item/309/2103.pdf?sequence=1\&isAllowed=y)

[59] Kočan et al. Rev. Sci. Instrum. 79, 0735022008

[60] Y. Peysson et al. Plasma Phys. Controlled Fusion, 54, 045003 (2012)

[61] C.C. Klepper et al. APS 2019 "Assessing the Impact of Light Impurities on Tungsten Sourcing Beyond the Divertor in WEST"

[62] G.Y. Antar et al 2012 Nucl. Fusion 52103005

[63] Jacquot J. et al 2015 AIP Conf. Proc. 1689050008

[64] D. Milanesio et al. 2009 Nucl. Fusion 49115019

[65] V. Lancellotti et al. 2006 Nucl. Fusion 46 S476

[66] W. Tierens et al 2019 Nucl. Fusion 59046001

[67] D.A. Pappas et al, J. Nucl. Mater. 266269 (1999) 635

[68] G. Urbanczyk et al. Nuclear Materials and Energy 26 (2021) 100925

[69] A. Gallo et al 2020 Nucl. Fusion 60126048

[70] Private communication with Arvidas Sepetys (http://www.theses.fr/2019AIXM0506)

[71] Wukitch et al. Phys. Plasmas 20, 056117 (2013)

[72] V. Bobkov et al. Nuclear Materials and Energy 12 (2017) 1194-1198

[73] Ragona R. and Messiaen A. 2016 Nucl. Fusion 56076009

[74] R. Ragona et al. AIP Conference Proceedings 2254, 070014 (2020) 


\section{Appendix}
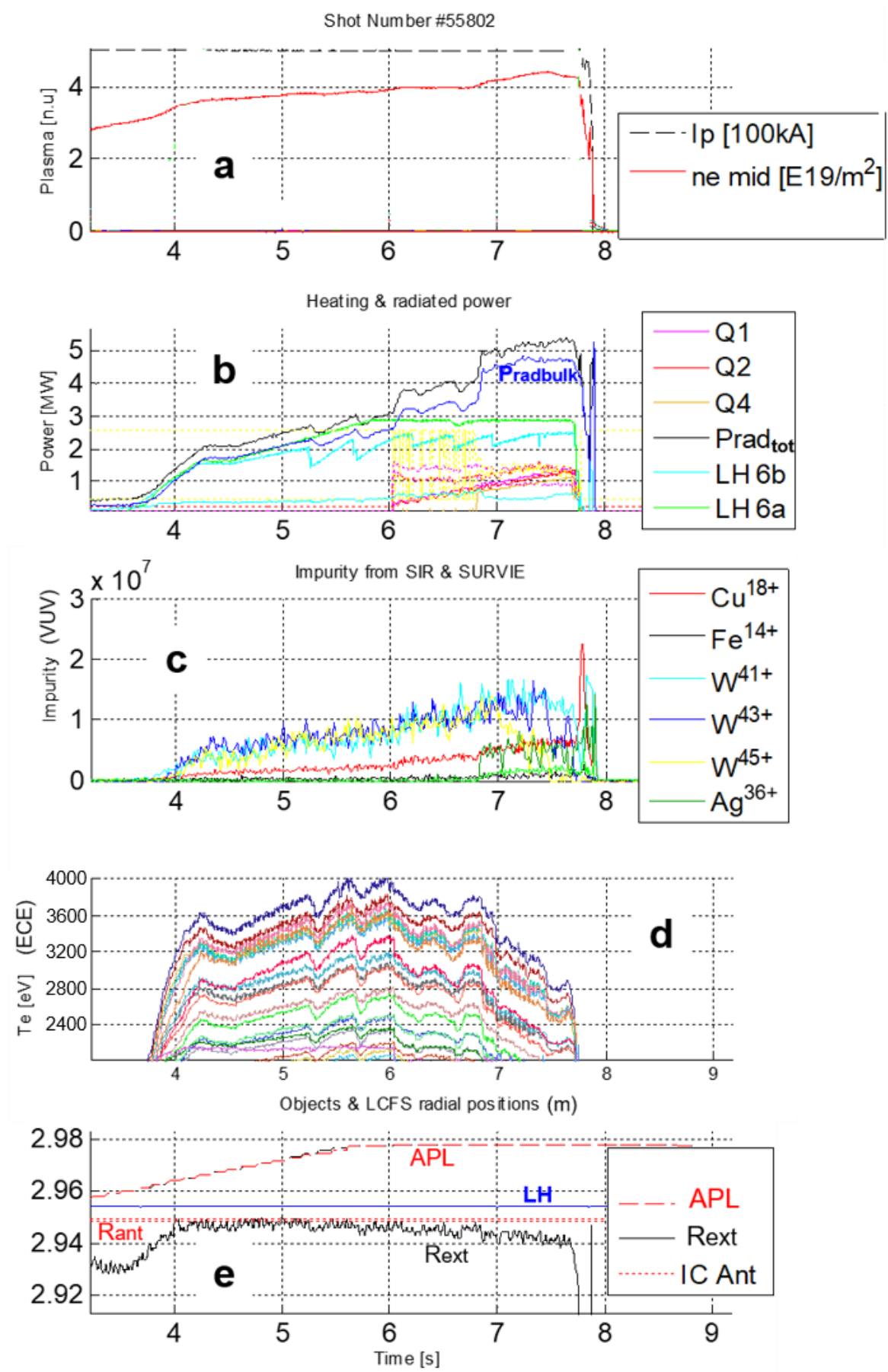

FIG A1: Overview of the discharge 55802 to which references are being made all along the paper and particularly in the last section: (a) Plasma current and electron density from a central chord of the interferometry (b) Power coupled from all antennas and radiated (c) Brightness of different high-Z impurity lines measured with VUV spectrometers (d) Electron temperature from all ECE lines (e)

Radial position at the midplane of different objects and the separatrix 


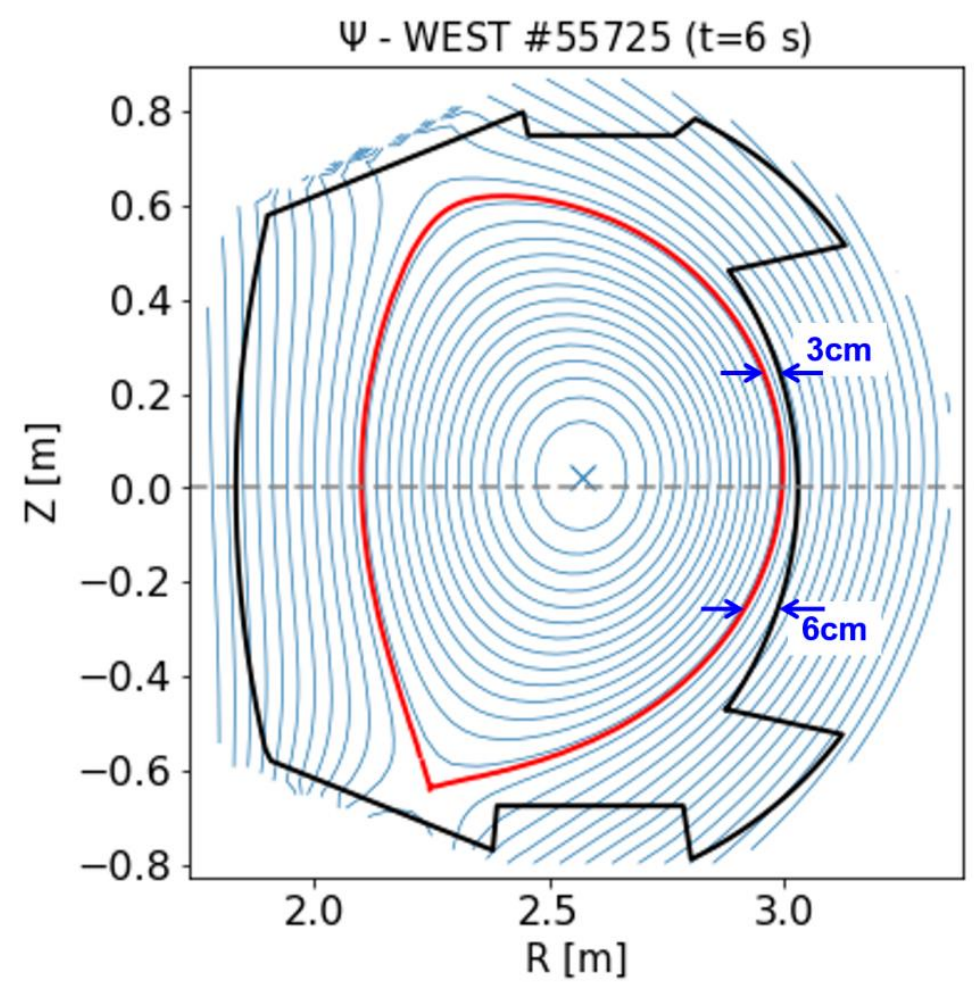

FIG A2: Poloidal cross section showing the magnetic equilibrium of the discharge 55725.

The distances between the separatrix (in red) and primary limiter (ROG) are materialized in blue, showing $3 \mathrm{~cm}$ at $Z=25 \mathrm{~cm}$ and $6 \mathrm{~cm}$ at $Z=-25 \mathrm{~cm}$, revealing the top-down asymmetry, typical of the wide majority of WEST discharges up to 2021 at least. 